\title{
A Nonlinear Liouville Property for the Generalized Kawahara Equation
}

\author{
André Kabakouala \\ People's Friendship University of Russia (RUDN University), \\ Miklukho-Maklaya str. 6, Moscow, 117198 Russian Federation. \\ kadodo1987@yahoo.fr
}

\begin{abstract}
In this paper, by applying the method of Martel and Merle [9, we prove that if the global solution of the generalized Kawahara equation ( $\mathrm{gKW}$ ) is close to the soliton (constructed by Kabakouala and Molinet [4]) at initial time in the energy space, moreover if this solution is uniformly localized, then it is identically equal to a soliton all time.
\end{abstract}

\section{Introduction}

The generalized Kawahara equation is given by:

$$
u_{t}+u^{p} \partial_{x} u+\partial_{x}^{3} u-\mu \partial_{x}^{5} u=0, \quad(t, x) \in \mathbb{R}_{+}^{*} \times \mathbb{R},
$$

where $p \in \mathbb{N}^{*}$ denotes the power of nonlinearity, and $\mu>0$ the parameter which control the fifth-order dispersion term. For $p=1$ and 2 , the gKW equation has applications for instance in fuid mechanics and plasma physics. For $p \geq 3$, what interests us is the balance between the nonlinear effect and the scattering effect, this leads to the formation of solitary waves.

The local Cauchy problem associated to 11.1 is locally well-posed in $H^{2}(\mathbb{R})$ (see for instance Abdelouhab et al. [1]). The following conservation laws hold for the $H^{2}(\mathbb{R})$ solutions:

$$
E_{p, \mu}(u(t))=\int_{\mathbb{R}}\left[\frac{\mu}{2}\left(\partial_{x}^{2} u\right)^{2}(t)+\frac{1}{2}\left(\partial_{x} u\right)^{2}(t)-\frac{1}{(p+1)(p+2)} u^{p+2}(t)\right]=E_{p, \mu}\left(u_{0}\right) \quad(\text { energy })
$$

and

$$
V(u(t))=\frac{1}{2} \int_{\mathbb{R}} u^{2}(t)=V\left(u_{0}\right) \quad(\text { mass }) .
$$

The global existence of all solutions in $H^{2}(\mathbb{R})$, as well as uniform bound in $H^{2}(\mathbb{R})$, follow from these conserved quantities. Moreover, thanks to the conservation laws one can rewrite equation (1.1) in the Hamiltonian form:

$$
\partial_{t} V^{\prime}(u)=\partial_{x} E^{\prime}(u)
$$

where $V^{\prime}(u)$ and $E^{\prime}(u)$ denote respectively the Fréchet derivative of $V(u)$ and $E(u)$.

The solitons of gKW are solution of 1.1) of the form $u(t, x)=\varphi_{c, p, \mu}(x-c t)$, which travels to the right direction with the constant speed $c>0$. Substituting $u$ by $\varphi_{c, p, \mu}$ in (1.1), integrating on $\mathbb{R}$ with 
the assumption $\partial_{x}^{k} \varphi_{c, p, \mu}( \pm \infty)=0$ for $k=0, \ldots 4$, we obtain the equation of $\mathrm{gKW}$-solitons:

$$
\mu \partial_{x}^{4} \varphi_{c, p, \mu}(x)-\partial_{x}^{2} \varphi_{c, p, \mu}(x)+c \varphi_{c, p, \mu}(x)=\frac{1}{p+1} \varphi_{c, p, \mu}^{p+1}(x), \quad \forall x \in \mathbb{R} .
$$

In 1996 Dey, Khare and Kumar [2 compute the explicit solitons of gKW, they found:

$$
\varphi_{c, p, \mu}(x)=\left[\frac{(p+1)(p+4)(3 p+4) c}{8(p+2)}\right]^{1 / p} \operatorname{sech}^{4 / p}\left[\frac{p \sqrt{\left(p^{2}+4 p+8\right) c}}{4(p+2)} x\right], \quad \forall x \in \mathbb{R},
$$

with a speed completely determined when we fix the value of $\mu$ :

$$
c=\frac{2^{2}(p+2)^{2}}{\left(p^{2}+4 p+8\right)^{2} \mu} .
$$

Due to the necessary condition of stability of the soliton $\varphi_{c, p, \mu}$ introduced by Karpman [5]:

$$
\frac{\mu \int_{\mathbb{R}}\left(\partial_{x}^{2} \varphi_{c, p, \mu}(x)\right)^{2}}{c \int_{\mathbb{R}} \varphi_{c, p, \mu}^{2}(x)}>\frac{p(p-4)}{\left(p^{2}+4 p+32\right)},
$$

Dey et al. 2] obtain that the single soliton $\varphi_{c, p, \mu}$ is unstable with respect to the small perturbation in the case $p \geq 5$.

Now we fix $\mu_{p}=\frac{2^{2}(p+2)^{2}}{\left(p^{2}+4 p+8\right)^{2}}$ then the speed of the explicit soliton 1.6$)-1.7$ is $c_{0}=1$. In Kabakouala and Molinet 4, by applying the Implicit Function Theorem in the neighborhood of $\varphi_{1, p, \mu_{p}}$, we construct a continuous in $H^{4}(\mathbb{R})$ branch $\left\{\varphi_{c_{0}, p, \mu_{p}}, c_{0} \in\right] 1-\delta_{p}, 1+\delta_{p}[\}$, with $0<\delta_{p} \ll 1$, of even solutions to equation (1.5). Moreover, for each $\left.c_{0} \in\right] 1-\delta_{p}, 1+\delta_{p}\left[, \varphi_{c_{0}, p, \mu_{p}}\right.$ is the unique even $H^{4}(\mathbb{R})$ solution of 1.5 in some $H^{4}$-neighborhood of $\varphi_{1, p, \mu_{p}}$ (see Lemma 2.2. Next, we prove the orbital stability of these family by combining the continuity arguments: for $c_{0}$ close to 1 , we have $\varphi_{c_{0}, p, \mu_{p}}$ is close to $\varphi_{1, p, \mu_{p}}$, and the well-known sufficient condition of the stability of the soliton $\varphi_{1, p, \mu_{p}}$ introduced by Bejamin: $\left\langle\mathcal{L}_{1, p, \mu_{p}}^{-1} \varphi_{1, p, \mu_{p}}, \varphi_{1, p, \mu_{p}}\right\rangle_{L^{2}}<0$, where $\mathcal{L}_{1, p, \mu_{p}} u=\mu_{p} \partial_{x}^{4} u-\partial_{x}^{2} u+u-\varphi_{1, p, \mu_{p}}^{p} u$. This stability condition has been verified numerically and is satisfied for the nonlinearity $p \in\{1,2,3,4\}$ (see [4] Subsection 2.3 and Subsection 2.4).

In a short note Kabakouala [3, by using the method of Martel 7 applied on the generalized Kortewegde Vries equation $(\mathrm{gKdV})$, we prove the linear Liouville property for the gKW equation: if $u(t, x)$ is solution to the linearized gKW equation around $\varphi_{c_{0}, p, \mu_{p}}: \partial_{t} u=\partial_{x}\left(\mathcal{L}_{c_{0}, p, \mu_{p}} u\right)$, where $\mathcal{L}_{c_{0}, p, \mu_{p}} u=\mu_{p} \partial_{x}^{4} u-$ $\partial_{x}^{2} u+c_{0} u-\varphi_{c_{0}, p, \mu_{p}}^{p} u$, and moreover if $u(t, x)$ is uniformly localized: $|u(t, x)| \lesssim e^{-\sigma|x|}$, then $u(t, x)$ is static and coincides with the unique element of the kernel of $\mathcal{L}_{c_{0}, p, \mu_{p}}$ which is $\varphi_{c_{0}, p, \mu_{p}}^{\prime}$.

In this paper, following the method of Martel and Merle [9] on the gKdV equation, we prove the nonlinear Liouville property for the gKW equation: if $u(t, x)$ is a $H^{2}$-global solution of gKW equation close to the soliton $\varphi_{c_{0}, p, \mu}$ (up to a translation) in the energy space: $\left\|u(t, \cdot+\xi(t))-\varphi_{c_{0}, p, \mu_{p}}\right\|_{H^{2}(\mathbb{R})} \leq \gamma$, with $\gamma \ll 1$, moreover if $u(t, x)$ is uniformly localized: $|u(t, x+\xi(t))| \leq e^{-\sigma|x|}$, then $u(t, x)$ is identically equal to a soliton $\varphi_{c_{1}, p, \mu_{p}}\left(x-\xi_{1}-c_{1} t\right)$, where $\left|c_{1}-c_{0}\right| \leq \gamma$ and $\left|\xi_{1}\right| \leq \gamma$.

Theorem 1.1 (Nonlinear Liouville property). Assume that $\left.p \in\{1,2\}, c_{0} \in\right] 1-\delta_{p}, 1+\delta_{p}\left[\right.$, with $\delta_{p} \ll 1$, and $\mu_{p}=\frac{2^{2}(p+2)^{2}}{\left(p^{2}+4 p+8\right)^{2}}$. There exists $\gamma_{0}>0$ such that if $0<\gamma<\gamma_{0}$ and $u \in C\left(\mathbb{R} ; H^{2}(\mathbb{R})\right)$ is a solution of $g K W$ equation satisfying for some function $\xi(t)$ and $\sigma>0$,

$$
\begin{gathered}
\left\|u(t, \cdot+\xi(t))-\varphi_{c_{0}, p, \mu_{p}}\right\|_{H^{2}(\mathbb{R})} \leq \gamma, \quad \forall t \in \mathbb{R}, \\
|u(t, x+\xi(t))| \lesssim e^{-\sigma|x|}, \quad \forall(t, x) \in \mathbb{R} \times \mathbb{R},
\end{gathered}
$$

then, there exist $c_{1}>0$ (close to 1 ) and $\xi_{1} \in \mathbb{R}$ (close to 0 ), such that

$$
u(t, x)=\varphi_{c_{1}, p, \mu_{p}}\left(x-\xi_{1}-c_{1} t\right), \quad \forall(t, x) \in \mathbb{R} \times \mathbb{R} .
$$

Remark 1.1 (Stability hypothesis). Due to the stability result of Kabakouala and Molinet 4, Theorem 1.1 remains true if we assume that: $\left\|u_{0}-\varphi_{c_{0}, p, \mu_{p}}\right\|_{H^{2}(\mathbb{R}} \leq \gamma$ instead of $[1.9$. 


\section{Nonlinear Liouville property}

\subsection{Preliminaries}

We start by recalling the spectral properties of the operator $\mathcal{L}_{1, p, \mu_{p}}$, the result of existence of a familly of solitons (solutions to (1.5) in a neighborhood of the explicit soliton $\varphi_{1, p, \mu_{p}}$ of velocity 1 (defined in (1.6)-(1.7), also we give the decomposition of the solution $u(t, x)$ around the soliton $\varphi_{c_{0}, p, \mu_{p}}$.

Lemma 2.1 (Spectral properties of $\mathcal{L}_{1, p, \mu_{p}}$; see [4). Let $p \in \mathbb{N}^{*}$. We consider the unbounded operator $\mathcal{L}_{1, p, \mu_{p}}: L^{2}(\mathbb{R}) \rightarrow L^{2}(\mathbb{R})$, defined by: $u \mapsto \mu_{p} \partial_{x}^{4} u-\partial_{x}^{2} u+u-\varphi_{1, p, \mu_{p}}^{p} u$. We claim that $\mathcal{L}_{1, p, \mu_{p}}$ possesses, among others, the following three crucial properties:

(P1) The essential spectrum of $\mathcal{L}_{1, p, \mu_{p}}$ is $[1,+\infty[$;

(P2) $\mathcal{L}_{1, p, \mu_{p}}$ has only one negative eigenvalue $\lambda_{1, p, \mu_{p}}$ which is simple;

(P3) The kernel of $\mathcal{L}_{1, p, \mu_{p}}$ is spanned by $\varphi_{1, p, \mu_{p}}^{\prime}$.

Lemma 2.2 (Existence of solitons $\varphi_{c_{0}, p, \mu_{p}}$ for $c_{0}$ close to 1 ; see [4]). There exist $\delta_{p}>0$ and $\tilde{\delta}_{p}>0$ such that for any $c_{0}>0$ with $\left|c_{0}-1\right|<\delta_{p}$, there exists a unique $H^{4}(\mathbb{R})$ even solution $\varphi_{c_{0}, p, \mu_{p}}$ of 1.5 in the ball of $H^{4}(\mathbb{R})$ centered at $\varphi_{1, p, \mu_{p}}$ with radius $\tilde{\delta}_{p}>0$. Moreover, the function $c_{0} \mapsto \varphi_{c_{0}, p, \mu_{p}}$ is of class $C^{1}$ from $] 1-\delta_{p}, 1+\delta_{p}\left[\right.$ into $B_{H^{4}}\left(\varphi_{1, p, \mu_{p}}\right)$.

Remark 2.1 (Important properties on $\varphi_{c_{0}, p, \mu_{p}}$ and $\mathcal{L}_{c_{0}, p, \mu_{p}}$ for $c_{0}$ close to 1 ; see [4] and [3]). We recall the following important properties and notations: $\varphi_{c_{0}, p, \mu_{p}} \in H^{k}(\mathbb{R})$ for all $k \in \mathbb{N} ;\left|\partial_{x}^{k} \varphi_{c_{0}, p, \mu_{p}}(x)\right| \lesssim e^{-\sqrt{c_{0}}|x|}$, for all $x \in \mathbb{R}$ and $\forall k \in \mathbb{N} ; \mathcal{L}_{c, p, \mu_{p}}\left(-\frac{d}{d c} \varphi_{c, p, \mu_{p}}\right)=\varphi_{c, p, \mu_{p}}$ and we set $\rho_{c_{0}, p, \mu_{p}}=-\left(\frac{d}{d c} \varphi_{c, p, \mu_{p}}\right)_{c=c_{0}}$; $\operatorname{ker}\left(\mathcal{L}_{c_{0}, p, \mu_{p}}\right)=\left\{0, \varphi_{c_{0}, p, \mu_{p}}^{\prime}\right\} ;\left\langle\mathcal{L}_{1, p, \mu_{p}}^{-1} \varphi_{1, p, \mu_{p}}, \varphi_{1, p, \mu_{p}}\right\rangle_{L^{2}}<0$ for $p \in\{1,2,3,4\} ;$ and $\left\langle\mathcal{L}_{1, p, \mu_{p}}^{-2} \varphi_{1, p, \mu_{p}}, \mathcal{L}_{1, p, \mu_{p}}^{-1} \varphi_{1, p, \mu_{p}}\right\rangle_{L^{2}}<$ 0 for $p \in\{1,2\}$.

Lemma 2.3 (Decomposition of $u$ around $\varphi_{c_{0}, p, \mu_{p}}$ ). Assume that $\left.p=\{1,2,3,4\}, c_{0} \in\right] 1-\delta_{p}, 1+\delta_{p}[$ with $\delta_{p} \ll 1$, and $\mu_{p}=\frac{2^{2}(p+2)^{2}}{\left(p^{2}+4 p+8\right)^{2}}$. There exist $\gamma_{0}>0$ and $K_{0}>0$ such that for $0<\gamma<\gamma_{0}$ the following is true. For any $u \in C\left(\mathbb{R} ; H^{2}(\mathbb{R})\right)$ solution of $g K W$ equation satisfying:

$$
\inf _{z \in \mathbb{R}}\left\|u(t, \cdot)-\varphi_{c_{0}, p, \mu_{p}}(\cdot-z)\right\|_{H^{2}(\mathbb{R})} \leq \gamma, \quad \forall t \in \mathbb{R},
$$

there exist $c(t) \in C^{1}(\mathbb{R})$ and $\xi(t) \in C^{1}(\mathbb{R})$ such that

$$
\eta(t, x)=u(t, x+\xi(t))-\varphi_{c(t), p, \mu_{p}}(x)
$$

satisfies for all $t \in \mathbb{R}$,

$$
\begin{gathered}
|c(t)-1|+\|\eta(t, \cdot)\|_{H^{2}(\mathbb{R})} \leq K_{0} \gamma, \\
\left\langle\eta(t, \cdot), \varphi_{c(t)}^{\prime}\right\rangle_{L^{2}}=\left\langle\eta(t, \cdot), \varphi_{c(t)}\right\rangle_{L^{2}}=0,
\end{gathered}
$$

and

$$
\left|c^{\prime}(t)\right|^{1 / 2}+\left|\xi^{\prime}(t)-c(t)\right| \leq K_{0}\|\eta\|_{L^{2}(\mathbb{R})} .
$$

Sketch of proof. The proof of Lemma 2.3 is a classical application of the Implicit Function Theorem (see for instance: Martel and Merle [8]). We just give the nondegeneracy conditions to satisfy the orthogonality conditions 2.4. Since $c_{0}$ is close to 1 , then by Lemma 2.2 we have

$$
\left\|\varphi_{c_{0}, p, \mu_{p}}^{\prime}\right\|_{L^{2}(\mathbb{R})}^{2} \sim\left\|\varphi_{1, p, \mu_{p}}^{\prime}\right\|_{L^{2}(\mathbb{R})}^{2}>0,
$$

and

$$
\left\langle\rho_{c_{0}, p, \mu_{p}}, \varphi_{c_{0}, p, \mu_{p}}\right\rangle_{L^{2}} \sim\left\langle\rho_{1, p, \mu_{p}}, \varphi_{1, p, \mu_{p}}\right\rangle_{L^{2}}<0 \text { for } 1 \leq p \leq 4,
$$

where $\rho_{c_{0}, p, \mu_{p}}$ is such that $\mathcal{L}_{c_{0}, p, \mu_{p}} \rho_{c_{0}, p, \mu_{p}}=\varphi_{c_{0}, p, \mu_{p}}$. 
Let us prove the control of modulation parameters $(2.5)$. We set $f(u)=[1 /(p+1)] u^{p+1}$ and we recall that $\rho_{c, p, \mu_{p}}=-\partial_{c} \varphi_{c, p, \mu_{p}}$. By differentiating (2.2) with respect to time and using equation of $u(t, x)$ and $\varphi_{c, p, \mu_{p}}$, we get the time variation of $\eta(t, x)$ :

$$
\begin{aligned}
\partial_{t} \eta & =\partial_{t} u+\xi^{\prime} \partial_{x} u+c^{\prime} \rho_{c, p, \mu_{p}} \\
& =\partial_{x}\left[\mu_{p} \partial_{x}^{4} \eta-\partial_{x}^{2} \eta+c \eta-\left(f\left(\varphi_{c, p, \mu_{p}}+\eta\right)-f\left(\varphi_{c, p, \mu_{p}}\right)\right)\right]+\left(\xi^{\prime}-c\right) \partial_{x}\left(\varphi_{c, p, \mu_{p}}+\eta\right)+c^{\prime} \rho_{c, p, \mu_{p}} \\
& =\partial_{x}\left[\mathcal{L}_{c, p, \mu_{p}} \eta-\left(f\left(\varphi_{c, p, \mu_{p}}+\eta\right)-f\left(\varphi_{c, p, \mu_{p}}\right)-f^{\prime}\left(\varphi_{c, p, \mu_{p}}\right) \eta\right)\right]+\left(\xi^{\prime}-c\right) \partial_{x}\left(\varphi_{c, p, \mu_{p}}+\eta\right)+c^{\prime} \rho_{c, p, \mu_{p}} .
\end{aligned}
$$

Now, differentiating equation $\left\langle\eta, \varphi_{c, p, \mu_{p}}\right\rangle_{L^{2}}=0$ with respect to time, using 2.8 and integration by parts, we have

$$
\begin{aligned}
& -\left\langle\mathcal{L}_{c, p, \mu_{p}} \eta-\left(f\left(\varphi_{c, p, \mu_{p}}+\eta\right)-f\left(\varphi_{c, p, \mu_{p}}\right)-f^{\prime}\left(\varphi_{c, p, \mu_{p}}\right) \eta\right), \varphi_{c, p, \mu_{p}}^{\prime}\right\rangle_{L^{2}} \\
& \quad-\left(\xi^{\prime}-c\right)\left\langle\varphi_{c, p, \mu_{p}}+\eta, \varphi_{c, p, \mu_{p}}^{\prime}\right\rangle_{L^{2}}+c^{\prime}\left\langle\varphi_{c, p, \mu_{p}}, \rho_{c, p, \mu_{p}}\right\rangle_{L^{2}}-c^{\prime}\left\langle\eta, \rho_{c, p, \mu_{p}}\right\rangle_{L^{2}}=0 .
\end{aligned}
$$

One can notice that

$$
f\left(\varphi_{c, p, \mu_{p}}+\eta\right)-f\left(\varphi_{c, p, \mu_{p}}\right)-f^{\prime}\left(\varphi_{c, p, \mu_{p}}\right) \eta=[1 /(p+1)] \sum_{k=2}^{p+1} C_{k}^{p+1} \varphi_{c, p, \mu_{p}}^{p-k+1} \eta^{k}
$$

Then, using that $\mathcal{L}_{c, p, \mu_{p}}\left(\varphi_{c, p, \mu_{p}}^{\prime}\right)=0,\left\langle\varphi_{c, p, \mu_{p}}+\eta, \varphi_{c, p, \mu_{p}}^{\prime}\right\rangle_{L^{2}}=0$, and combining 2.3, 2.7), 2.9) and (2.10), we obtain

$$
\begin{aligned}
\left|c^{\prime}\right| & \leq \frac{\frac{p}{2}\left|\left\langle\varphi_{c, p, \mu_{p}}^{p-1} \varphi_{c, p, \mu_{p}}^{\prime}, \eta^{2}\right\rangle_{L^{2}}\right|+[1 /(p+1)]\left|\sum_{k=3}^{p+1} C_{k}^{p+1}\left\langle\varphi_{c, p, \mu_{p}}^{p-k+1} \varphi_{c, p, \mu_{p}}^{\prime}, \eta^{k}\right\rangle_{L^{2}}\right|}{\left|\left\langle\varphi_{c, p, \mu_{p}}, \rho_{c, p, \mu_{p}}\right\rangle_{L^{2}}-\left\langle\eta, \rho_{c, p, \mu_{p}}\right\rangle_{L^{2}}\right|} \\
& \leq \frac{\left(\frac{p}{2}\left\|\varphi_{c, p, \mu_{p}}\right\|_{L^{\infty}(\mathbb{R})}^{p-1}\left\|\varphi_{c, p, \mu_{p}}^{\prime}\right\|_{L^{\infty}(\mathbb{R})}+K_{0} \gamma_{0}\right)\|\eta\|_{L^{2}(\mathbb{R})}^{2}}{-\left\langle\varphi_{c, p, \mu_{p}}, \rho_{c, p, \mu_{p}}\right\rangle_{L^{2}}-K_{0} \gamma_{0}} \\
& \leq K_{0}\|\eta\|_{L^{2}(\mathbb{R})}^{2} .
\end{aligned}
$$

Similarly, differentiating equation $\left\langle\eta, \varphi_{c, p, \mu_{p}}^{\prime}\right\rangle_{L^{2}}=0$ with respect to time, using 2.8 and integration by parts, we have

$$
\begin{aligned}
& -\left\langle\mathcal{L}_{c, p, \mu_{p}} \eta-\left(f\left(\varphi_{c, p, \mu_{p}}+\eta\right)-f\left(\varphi_{c, p, \mu_{p}}\right)-f^{\prime}\left(\varphi_{c, p, \mu_{p}}\right) \eta\right), \varphi_{c, p, \mu_{p}}^{\prime \prime}\right\rangle_{L^{2}} \\
& \quad-\left(\xi^{\prime}-c\right)\left\langle\left(\varphi_{c, p, \mu_{p}}+\eta\right), \varphi_{c, p, \mu_{p}}^{\prime \prime}\right\rangle_{L^{2}}+c^{\prime}\left\langle\varphi_{c, p, \mu_{p}}^{\prime}, \rho_{c, p, \mu_{p}}\right\rangle_{L^{2}}-c^{\prime}\left\langle\eta, \rho_{c, p, \mu_{p}}^{\prime}\right\rangle_{L^{2}}=0 .
\end{aligned}
$$


Next, using that $\left\langle\varphi_{c, p, \mu_{p}}^{\prime}, \rho_{c, p, \mu_{p}}\right\rangle_{L^{2}}=0,2.3,2.6$ and 2.11, we get

$$
\begin{aligned}
&\left|\xi^{\prime}-c\right| \leq \frac{\left|\left\langle\eta, \mathcal{L}_{c, p, \mu_{p}} \varphi_{c, p, \mu_{p}}^{\prime \prime}\right\rangle_{L^{2}}\right|+\left|c^{\prime}\left\langle\eta, \rho_{c, p, \mu_{p}}^{\prime}\right\rangle_{L^{2}}\right|}{\left|\left\langle\varphi_{c, p, \mu_{p}}+\eta, \varphi_{c, p, \mu_{p}}^{\prime \prime}\right\rangle_{L^{2}}\right|} \\
&+\frac{[1 /(p+1)] \sum_{k=2}^{p+1} C_{k}^{p+1}\left|\left\langle\varphi_{c, p, \mu_{p}}^{p-k+1} \varphi_{c, p, \mu_{p}}^{\prime \prime}, \eta^{k}\right\rangle_{L^{2}}\right|}{\left|\left\langle\varphi_{c, p, \mu_{p}}+\eta, \varphi_{c, p, \mu_{p}}^{\prime \prime}\right\rangle_{L^{2}}\right|} \\
& \leq \frac{\left\|\mathcal{L}_{c, p, \mu_{p}} \varphi_{c, p, \mu_{p}}^{\prime \prime}\right\|_{L^{2}(\mathbb{R})}\|\eta\|_{L^{2}(\mathbb{R})}+\left|c^{\prime}\right|\left\|\rho_{c, p, \mu_{p}}^{\prime}\right\|_{L^{2}(\mathbb{R})}\|\eta\|_{L^{2}(\mathbb{R})}}{\left\|\varphi_{c, p, \mu_{p}}^{\prime}\right\|_{L^{2}(\mathbb{R})}^{2}-K_{0} \gamma_{0}} \\
& \quad+\frac{\left(\frac{p}{2}\left\|\varphi_{c, p, \mu_{p}}\right\|_{L^{\infty}(\mathbb{R})}^{p-1}\left\|\varphi_{c, p, \mu_{p}}^{\prime \prime}\right\|_{L^{\infty}(\mathbb{R})}+K_{0} \gamma_{0}\right)\|\eta\|_{L^{2}(\mathbb{R})}^{2}}{\left\|\varphi_{c, p, \mu_{p}}^{\prime}\right\|_{L^{2}(\mathbb{R})}^{2}-K_{0} \gamma_{0}} \\
& \leq K_{0}\|\eta\|_{L^{2}(\mathbb{R})},
\end{aligned}
$$

and this prove 2.5 .

Lemma 2.4 (Smoothness and Exponential Decay). Let $u \in C\left(\mathbb{R} ; H^{2}(\mathbb{R})\right)$ be a solution of $g K W$ equation satisfying 2.1)-(2.5) and the decay property (1.10). Then $u \in C^{\infty}(\mathbb{R} \times \mathbb{R})$. Moreover, there exists $\sigma>0$ such that for all $k \in \mathbb{N}$,

$$
\left|\partial_{x}^{k} u(t, x+\xi(t))\right| \lesssim e^{-\sigma|x|}, \quad \forall(t, x) \in \mathbb{R} \times \mathbb{R} .
$$

The proof of Lemma 2.4 is based on a monotonicity property and is given in the Subsection 2.3

\subsection{Proof of Theorem 1.1}

We divide the proof in four steps and we argue as Martel and Merle 9 .

Step 1. Nonlinear dual problem related to gKW equation.

We set

$$
\begin{aligned}
v & =\mu_{p} \partial_{x}^{4} \eta-\partial_{x}^{2} \eta+c \eta-\left(f\left(\varphi_{c, p, \mu_{p}}+\eta\right)-f\left(\varphi_{c, p, \mu_{p}}\right)\right) \\
& =\mathcal{L}_{c, p, \mu_{p}} \eta-\left(f\left(\varphi_{c, p, \mu_{p}}+\eta\right)-f\left(\varphi_{c, p, \mu_{p}}\right)-f^{\prime}\left(\varphi_{c, p, \mu_{p}}\right) \eta\right) .
\end{aligned}
$$

By differentiating $v(x, t)$ with respect to $x$, we get

$$
\partial_{x} v=\mu_{p} \partial_{x}^{5} \eta-\partial_{x}^{3} \eta+c \partial_{x} v-\partial_{x} \eta f^{\prime}\left(\varphi_{c, p, \mu_{p}}+\eta\right)-\varphi_{c, p, \mu_{p}}^{\prime}\left(f^{\prime}\left(\varphi_{c, p, \mu_{p}}+\eta\right)-f^{\prime}\left(\varphi_{c, p, \mu_{p}}\right)\right) .
$$

Now, using (2.8), the time variation of $v(x, t)$ gives us

$$
\begin{aligned}
\partial_{t} v= & \mu_{p} \partial_{x}^{4} \eta_{t}-\partial_{x}^{2} \eta_{t}+c \eta_{t}-\eta_{t} f^{\prime}\left(\varphi_{c, p, \mu_{p}}+\eta\right)+c^{\prime} \eta+c^{\prime} \rho_{c, p, \mu_{p}}\left(f^{\prime}\left(\varphi_{c, p, \mu_{p}}+\eta\right)-f^{\prime}\left(\varphi_{c, p, \mu_{p}}\right)\right) \\
= & \mu_{p} \partial_{x}^{5} v-\partial_{x}^{3} v+c \partial_{x} v-\partial_{x} v f^{\prime}\left(\varphi_{c, p, \mu_{p}}+\eta\right) \\
& +\left(\xi^{\prime}-c\right)\left(\mu_{p} \partial_{x}^{5}\left(\varphi_{c, p, \mu_{p}}+\eta\right)-\partial_{x}^{3}\left(\varphi_{c, p, \mu_{p}}+\eta\right)+c \partial_{x}\left(\varphi_{c, p, \mu_{p}}+\eta\right)-\partial_{x}\left(\varphi_{c, p, \mu_{p}}+\eta\right) f^{\prime}\left(\varphi_{c, p, \mu_{p}}+\eta\right)\right) \\
& \quad+c^{\prime}\left(\mu_{p} \partial_{x}^{4} \rho_{c, p, \mu_{p}}-\partial_{x}^{2} \rho_{c, p, \mu_{p}}+c \rho_{c, p, \mu_{p}}-\rho_{c, p, \mu_{p}} f^{\prime}\left(\varphi_{c, p, \mu_{p}}\right)\right)+c^{\prime} \eta .
\end{aligned}
$$

Then, using that $\mathcal{L}_{c, p, \mu_{p}} \rho_{c, p, \mu_{p}}=\varphi_{c, p, \mu_{p}}, \mathcal{L}_{c, p, \mu_{p}} \varphi_{c, p, \mu_{p}}^{\prime}=0$ and 2.16, it holds

$$
\partial_{t} v=\mathcal{L}_{c, p, \mu_{p}}\left(\partial_{x} v\right)-\partial_{x} v\left(f^{\prime}\left(\varphi_{c, p, \mu_{p}}+\eta\right)-f^{\prime}\left(\varphi_{c, p, \mu_{p}}\right)\right)+\left(\xi^{\prime}-c\right) \partial_{x} v+c^{\prime}\left(\varphi_{c, p, \mu_{p}}+\eta\right) .
$$

Step 2. Almost orthogonality conditions of $v(t, x)$ with respect to $\varphi_{1, p, \mu_{p}}^{\prime}(x)$ and $\rho_{1, p, \mu_{p}}(x)$, and positivity property of the quadratic form $\mathcal{L}_{c, p, \mu_{p}}$ with respect to $v(t, x)$. 
We start with the almost orthogonality conditions. Using that $\mathcal{L}_{c, p, \mu_{p}} \varphi_{c, p, \mu_{p}}^{\prime}=0,2.10$ and 2.15, we have

$$
\begin{aligned}
\left|\left\langle v, \varphi_{c, p, \mu_{p}}^{\prime}\right\rangle_{L^{2}}\right| & =\left|\left\langle\mathcal{L}_{c, p, \mu_{p}} \eta-\left(f\left(\varphi_{c, p, \mu_{p}}+\eta\right)-f\left(\varphi_{c, p, \mu_{p}}\right)-f^{\prime}\left(\varphi_{c, p, \mu_{p}}\right) \eta\right), \varphi_{c, p, \mu_{p}}^{\prime}\right\rangle_{L^{2}}\right| \\
& \leq\left(\frac{p}{2}\left\|\varphi_{c, p, \mu_{p}}\right\|_{L^{\infty}(\mathbb{R})}^{p-1}\left\|\varphi_{c, p, \mu_{p}}^{\prime}\right\|_{L^{\infty}(\mathbb{R})}+K_{0} \gamma_{0}\right)\|\eta\|_{L^{2}(\mathbb{R})}^{2} \\
& \leq K_{0}\|\eta\|_{L^{2}(\mathbb{R})}^{2} .
\end{aligned}
$$

Next, using $\left\langle\eta, \varphi_{c, p, \mu_{p}}\right\rangle_{L^{2}}=0, \mathcal{L}_{c, p, \mu_{p}} \rho_{c, p, \mu_{p}}=\varphi_{c, p, \mu_{p}}$, 2.10 and 2.15, we have

$$
\begin{aligned}
\left|\left\langle v, \rho_{c, p, \mu_{p}}\right\rangle_{L^{2}}\right| & =\left|\left\langle\mathcal{L}_{c, p, \mu_{p}} \eta-\left(f\left(\varphi_{c, p, \mu_{p}}+\eta\right)-f\left(\varphi_{c, p, \mu_{p}}\right)-f^{\prime}\left(\varphi_{c, p, \mu_{p}}\right) \eta\right), \rho_{c, p, \mu_{p}}\right\rangle_{L^{2}}\right| \\
& \leq\left(\frac{p}{2}\left\|\varphi_{c, p, \mu_{p}}\right\|_{L^{\infty}(\mathbb{R})}^{p-1}\left\|\rho_{c, p, \mu_{p}}\right\|_{L^{\infty}(\mathbb{R})}+K_{0} \gamma_{0}\right)\|\eta\|_{L^{2}(\mathbb{R})}^{2} \\
& \leq K_{0}\|\eta\|_{L^{2}(\mathbb{R})}^{2} .
\end{aligned}
$$

From (2.4), we know that $\eta \in\left\{\varphi_{c, p, \mu_{p}}^{\prime}, \varphi_{c, p, \mu_{p}}\right\}^{\perp}$, then $\left|\left\langle\eta, \varphi_{1, p, \mu_{p}}^{\prime}\right\rangle_{L^{2}}\right|+\left|\left\langle\eta, \varphi_{1, p, \mu_{p}}\right\rangle_{L^{2}}\right| \lesssim \gamma_{0}\|\eta\|_{L^{2}(\mathbb{R})}$ (because $|c-1| \leq \gamma_{0}$ ). Since $\left\langle\rho_{1, p, \mu_{p}}, \varphi_{1, p, \mu_{p}}\right\rangle_{L^{2}}<0$ for $1 \leq p \leq 4$, arguing as Kabakouala and Molinet [4] (see Subsections 2.3 and 2.4), we infer that there exists $C_{0}>0$ such that

$$
\left\langle\mathcal{L}_{c, p, \mu_{p}} \eta, \eta\right\rangle_{L^{2}} \sim\left\langle\mathcal{L}_{1, p, \mu_{p}} \eta, \eta\right\rangle_{L^{2}} \geq C_{0}\|\eta\|_{L^{2}(\mathbb{R})}^{2} .
$$

Now, combining (2.3) and (2.21), we have

$$
\begin{aligned}
\langle v, \eta\rangle_{L^{2}} & =\left\langle\mathcal{L}_{c, p, \mu_{p}} \eta, \eta\right\rangle_{L^{2}}-\left\langle f\left(\varphi_{c, p, \mu_{p}}+\eta\right)-f\left(\varphi_{c, p, \mu_{p}}\right)-f^{\prime}\left(\varphi_{c, p, \mu_{p}}\right) \eta, \eta\right\rangle_{L^{2}} \\
& \geq C_{0}\|\eta\|_{L^{2}(\mathbb{R})}^{2}-\left(\frac{p}{2}\left\|\varphi_{c, p, \mu_{p}}\right\|_{L^{\infty}(\mathbb{R})}^{p-1}+K_{0} \gamma_{0}\right)\|\eta\|_{L^{3}(\mathbb{R})}^{3} \\
& \geq K_{0}\|\eta\|_{L^{2}(\mathbb{R})}^{2} .
\end{aligned}
$$

Thus, applying Cauchy-Schwarz inequality to $\langle v, \eta\rangle_{L^{2}}$ and using 2.22 , we obtain

$$
\|\eta\|_{L^{2}(\mathbb{R})} \leq K_{0}\|v\|_{L^{2}(\mathbb{R})} .
$$

Finally, comnining (2.19)-2.20) and 2.23), and using that by continuity $\varphi_{c, p, \mu_{p}}$ (respectively $\left.\rho_{c, p, \mu_{p}}\right)$ is close to $\varphi_{1, p, \mu_{p}}$ in $H^{4}(\mathbb{R})$ (respectively to $\rho_{c, p, \mu_{p}}$ ) when $c$ is close to 1 , we infer that $\left|\left\langle v, \varphi_{1, p, \mu_{p}}^{\prime}\right\rangle_{L^{2}}\right|+$ $\left|\left\langle v, \rho_{1, p, \mu_{p}}\right\rangle_{L^{2}}\right| \lesssim \gamma_{0}\|v\|_{L^{2}(\mathbb{R})}$. Since $\left\langle\mathcal{L}_{1, p, \mu_{p}}^{-1} \rho_{1, p, \mu_{p}}, \rho_{1, p, \mu_{p}}\right\rangle<0$ for $1 \leq p \leq 2$, once again, proceeding as Kabakouala and Molinet [4] (see Subsection 2.3 and 2.4), we deduce that there exists $C_{1}>0$ such that

$$
\left\langle\mathcal{L}_{c, p, \mu_{p}} v, v\right\rangle_{L^{2}} \sim\left\langle\mathcal{L}_{1, p, \mu_{p}} v, v\right\rangle_{L^{2}} \geq C_{1}\|v\|_{L^{2}(\mathbb{R})}^{2} .
$$

Step 3. Virial identity related to 2.18).

By multiplying (2.18) with the function $v(t, x) x$, and using the computations done on the Linear Dual Problem (see Kabakouala [3, Subsection 2.2, p. $5-6$ ), we establish that

$$
\begin{aligned}
-\frac{d}{d t} \int_{\mathbb{R}} v^{2} x & =-2 \int_{\mathbb{R}} \mathcal{L}_{c, p, \mu_{p}}\left(\partial_{x} v\right) v x+\mathcal{R}(\eta, v) \\
& =5 \mu_{p} \int_{\mathbb{R}}\left(\partial_{x}^{2} v\right)^{2}+3 \int_{\mathbb{R}}\left(\partial_{x} v\right)^{2}+c \int_{\mathbb{R}} v^{2}-\int_{\mathbb{R}} v^{2} \varphi_{c, p, \mu_{p}}^{p}-p \int_{\mathbb{R}} v^{2} \varphi_{c, p, \mu_{p}}^{p-1} \varphi_{c, p, \mu_{p}}^{\prime} x+\mathcal{R}(\eta, v),
\end{aligned}
$$

with

$$
\begin{gathered}
\mathcal{R}(\eta, v)=-\int_{\mathbb{R}} v^{2}\left(f^{\prime}\left(\varphi_{c, p, \mu_{p}}+\eta\right)-f^{\prime}\left(\varphi_{c, p, \mu_{p}}\right)\right)-\int_{\mathbb{R}} v^{2}\left(f^{\prime \prime}\left(\varphi_{c, p, \mu_{p}}+\eta\right)-f^{\prime \prime}\left(\varphi_{c, p, \mu_{p}}\right)\right) \varphi_{c, p, \mu_{p}}^{\prime} x \\
-\int_{\mathbb{R}} v^{2} f^{\prime \prime}\left(\varphi_{c, p, \mu_{p}}+\eta\right) \eta^{\prime} x+\left(\xi^{\prime}-c\right) \int_{\mathbb{R}} v^{2}-2 c^{\prime} \int_{\mathbb{R}} v\left(\varphi_{c, p, \mu_{p}}+\eta\right) x .
\end{gathered}
$$


Let us estimate the term $\mathcal{R}(v, \eta)$. Using that $\|\eta\|_{C^{1}(\mathbb{R})} \leq C_{S}\|\eta\|_{H^{2}(\mathbb{R})}$ (by Sobolev embedding), $f^{\prime}\left(\varphi_{c, p, \mu_{p}}+\right.$ $\eta)-f^{\prime}\left(\varphi_{c, p, \mu_{p}}\right)=\sum_{k=1}^{p} C_{k}^{p} \varphi_{c, p, \mu_{p}}^{p-k} \eta^{k}=0,2.5$ and 2.23, we have the following estimates:

$$
\begin{aligned}
\left|\int_{\mathbb{R}} v^{2}\left(f^{\prime}\left(\varphi_{c, p, \mu_{p}}+\eta\right)-f^{\prime}\left(\varphi_{c, p, \mu_{p}}\right)\right)\right| & \leq\|v\|_{L^{2}(\mathbb{R})}^{2} \sum_{k=1}^{p} C_{k}^{p}\left\|\eta^{k}\right\|_{L^{\infty}(\mathbb{R})}\left\|\varphi_{c, p, \mu_{p}}^{p-k}\right\|_{L^{\infty}(\mathbb{R})} \\
& \leq K_{0}\|\eta\|_{L^{2}(\mathbb{R})}\|v\|_{H^{2}(\mathbb{R})}^{2}
\end{aligned}
$$

and

$$
\begin{gathered}
\left|\left(\xi^{\prime}-c\right) \int_{\mathbb{R}} v^{2}\right| \leq K_{0}\|\eta\|_{L^{2}(\mathbb{R})}\|v\|_{L^{2}(\mathbb{R})}^{2}, \\
\left|c^{\prime} \int_{\mathbb{R}} v\left(\varphi_{c, p, \mu_{p}}+\eta\right) x\right| \leq\left|c^{\prime}\right|\left\|\left(\varphi_{c, p, \mu_{p}}+\eta\right) x\right\|_{L^{2}(\mathbb{R})}\|v\|_{L^{2}(\mathbb{R})} \\
\leq K_{0}\|\eta\|_{L^{2}(\mathbb{R})}\|v\|_{L^{2}(\mathbb{R})}^{2} .
\end{gathered}
$$

Similarly for the other terms. Thus, we deduce that

$$
|\mathcal{R}(v, \eta)| \leq K_{0}\|\eta\|_{L^{2}(\mathbb{R})}\|v\|_{H^{2}(\mathbb{R})}^{2} .
$$

Step 4. Conclusion of the proof of Theorem 1.1 .

At this stage, we have all the ingredients to prove Theorem 1.1. First, using the definition of $\mathcal{L}_{c, p, \mu_{p}}$, one can rewrite identity 2.25 as

$$
-\frac{d}{d t} \int_{\mathbb{R}} v^{2} x=\left\langle\mathcal{L}_{c, p, \mu_{p}} v, v\right\rangle_{L^{2}}+4 \mu_{p} \int_{\mathbb{R}}\left(\partial_{x}^{2} v\right)^{2}+2 \int_{\mathbb{R}}\left(\partial_{x} v\right)^{2}-p \int_{\mathbb{R}} v^{2} \varphi_{c, p, \mu_{p}}^{p-1} \varphi_{c, p, \mu_{p}}^{\prime} x+\mathcal{R}(v, \eta) .
$$

With the last integral we deal as follow, let us set $\varphi_{c_{0}, p, \mu_{p}}(x)=\varphi_{1, p, \mu_{p}}(x)+\left(\varphi_{c_{0}, p, \mu_{p}}(x)-\varphi_{1, p, \mu_{p}}(x)\right)=$ $\varphi_{c_{0}, p, \mu_{p}}(x)+\gamma(x)$. For $c_{0}$ close to 1 , we have $\sup _{x \in \mathbb{R}}|\gamma(x)|+\sup _{x \in \mathbb{R}}\left|\gamma^{\prime}(x)\right| \leq \gamma$, with $0<\gamma \ll 1$, and $|\gamma(x)|+\left|\gamma^{\prime}(x)\right| \lesssim e^{-\sqrt{\min \left(c_{0}, 1\right)}|x|}$ for all $x \in \mathbb{R}$. Then we deduce the following estimate:

$$
-p \int_{\mathbb{R}} v^{2} \varphi_{c_{0}, p, \mu_{p}}^{p-1} \varphi_{c_{0}, p, \mu_{p}}^{\prime} x \geq-p \int_{\mathbb{R}} v^{2} \varphi_{1, p, \mu_{p}}^{p-1} \varphi_{1, p, \mu_{p}}^{\prime} x-\gamma \int_{\mathbb{R}} v^{2} .
$$

Since $\varphi_{c_{0}, p, \mu_{p}}(x)>0$ and $-\varphi_{c_{0}, p, \mu_{p}}^{\prime}(x) x>0$ for all $x \in \mathbb{R}$, we get

$$
-p \int_{\mathbb{R}} v^{2} \varphi_{c_{0}, p, \mu_{p}}^{p-1} \varphi_{c_{0}, p, \mu_{p}}^{\prime} x \geq-\gamma \int_{\mathbb{R}} v^{2} .
$$

Next, combining 2.3, 2.24, 2.30, 2.31) and 2.33), we infer the following inequality:

$$
-\frac{d}{d t} \int_{\mathbb{R}} v^{2} x \geq\left(C_{1}-K_{0} \gamma_{0}\right)\|v\|_{H^{2}(\mathbb{R})}^{2} \geq C_{2}\|v\|_{H^{2}(\mathbb{R})}^{2} .
$$

Now, Lemma 2.4 the definition of $\mathcal{L}_{c_{0}, p, \mu_{p}}$, the smoothness and exponential decay properties of $\varphi_{c_{0}, p, \mu_{p}}$, imply that $v \in C^{\infty}(\mathbb{R} \times \mathbb{R})$, and there exists $\sigma_{0}>0$ such that

$$
|v(t, x)| \lesssim e^{-\sigma_{0}|x|}, \quad \forall(t, x) \in \mathbb{R} \times \mathbb{R} .
$$

For all $t \in \mathbb{R}$, we set

$$
\mathcal{J}(t)=\int_{\mathbb{R}} v^{2}(t, x) x .
$$

Thanks to 2.35), one can check that for all $t \in \mathbb{R}$,

$$
|\mathcal{J}(t)| \leq \int_{\mathbb{R}} v^{2}(t, x)|x| \lesssim \int_{\mathbb{R}} e^{-2 \sigma|x|}|x|=\frac{1}{2 \sigma_{1}^{2}} .
$$


Now, integrating 2.34 with respect to time and using 2.37), we obtain

$$
\int_{-\infty}^{+\infty}\|v(t)\|_{H^{2}(\mathbb{R})}^{2} d t \leq C_{1}^{-1}(\mathcal{J}(-\infty)-\mathcal{J}(+\infty))<+\infty
$$

It follows that for a time sequence $t_{n} \rightarrow+\infty$, we have $v\left(t_{n}\right) \rightarrow 0$ in $H^{2}(\mathbb{R})$. In particular, we have

$$
\left|\mathcal{J}\left(t_{n}\right)\right| \leq\left\|v\left(t_{n}\right) x\right\|_{L^{2}(\mathbb{R})}\left\|v\left(t_{n}\right)\right\|_{L^{2}(\mathbb{R})} \lesssim \frac{1}{\sqrt{2 \sigma^{3}}}\left\|v\left(t_{n}\right)\right\|_{L^{2}(\mathbb{R})} \rightarrow 0 \text { as } t_{n} \rightarrow+\infty .
$$

We infer that $\mathcal{J}(+\infty)=0$. Similarly, $\mathcal{J}(-\infty)=0$. Thus 2.38 becomes

$$
\int_{-\infty}^{+\infty}\|v(t)\|_{H^{2}(\mathbb{R})}^{2} d t=0
$$

which implies that $v(t, x) \equiv 0$ for all $(t, x) \in \mathbb{R} \times \mathbb{R}$. Then, from $(2.23)$, we infer that $\eta(t, x)=0$, and from (2.5), we have $c(t)=c(0)$ and $\xi(t)=\xi(0)+c(0) t$. We set $c(0)=c_{1}$ and $\xi(0)=\xi_{1}$, thus $(2.2)$ leads to $u(t, x)=\varphi_{c_{1}}\left(x-\xi_{1}-c_{1} t\right)$ for all $(t, x) \in \mathbb{R} \times \mathbb{R}$. This proves 1.11 .

\subsection{Monotonicity property related to the gKW equation}

In this section we give the proof of Lemma 2.4. We follows the arguments of Laurent and Martel [6] with the gKdV equation, and we present the proof in six steps.

We consider the same test function: $\phi(x)=\frac{1}{2}+\frac{1}{2} \tanh \left(\frac{x}{2}\right)$ for $x \in \mathbb{R}$, as in Kabakouala [3] (Subsection 2.3). One can easy check the following properties: $\phi^{\prime}(x)=\frac{1}{4} \operatorname{sech}^{2}\left(\frac{x}{2}\right)$ and $\left|\phi^{(j+1)}(x)\right| \lesssim \phi^{\prime}(x)$, for all $j \in \mathbb{N}^{*}$, and for all $x \in \mathbb{R}$. Let $K>0$, we set $\phi_{K}(x)=\phi(x / K)$. Then we deduce that $\left|\phi_{K}^{(j+1)}(x)\right| \leq \frac{1}{K^{j}} \phi_{K}^{\prime}(x)$.

Let $x_{0}>0$ and $t_{0} \in \mathbb{R}$. For all $t \leq t_{0}$, we set $\bar{x}=x-\xi\left(t_{0}\right)+\frac{c_{0}}{2}\left(t_{0}-t\right)-x_{0}$, and we define the following $L^{2}$-localized energy:

$$
I_{x_{0}, t_{0}}(t)=\int_{\mathbb{R}} u^{2}(t, x) \phi_{K}(\bar{x}) d x .
$$

We start with the almost monotonicity property of the functional $I_{x_{0}, t_{0}}(t)$.

Step 1. For $x_{0}>0$ and $t \leq t_{0}$, we claim that:

$$
I_{x_{0}, t_{0}}\left(t_{0}\right)-I_{x_{0}, t_{0}}(t) \lesssim e^{-x_{0} / K} .
$$

Proof of 2.41). Applying the Virial Type Identity I (see Subsection 2.4), the time variation of $I_{x_{0}, t_{0}}(t)$ leads to

$$
\begin{aligned}
\frac{d}{d t} I_{x_{0}, t_{0}}(t)= & -5 \mu_{p} \int_{\mathbb{R}}\left(\partial_{x}^{2} u\right)^{2} \phi_{K}^{\prime}(\bar{x})-3 \int_{\mathbb{R}}\left(\partial_{x} u\right)^{2} \phi_{K}^{\prime}(\bar{x})-\frac{c_{0}}{2} \int_{\mathbb{R}} u^{2} \phi_{K}^{\prime}(\bar{x}) \\
& +5 \mu_{p} \int_{\mathbb{R}}\left(\partial_{x} u\right)^{2} \phi_{K}^{\prime \prime \prime}(\bar{x})-\mu_{p} \int_{\mathbb{R}} u^{2} \phi_{K}^{(5)}(\bar{x})+\int_{\mathbb{R}} u^{2} \phi_{K}^{\prime \prime \prime}(\bar{x}) \\
& +\frac{2}{p+2} \int_{\mathbb{R}} u^{p+2} \phi_{K}^{\prime}(\bar{x}) .
\end{aligned}
$$

Note that the negative term: $-5 \mu_{p} \int_{\mathbb{R}}\left(\partial_{x}^{2} u\right)^{2} \phi^{\prime}(\bar{x})$ will permit us to gain regularity in the next steps.

Let us make the estimate of the terms in the second line of $\left(2.42\right.$. Using that $\left|\phi_{K}^{(j+1)}\right| \lesssim \frac{1}{K^{j}} \phi_{K}^{\prime}$ on $\mathbb{R}$, for $j=1, \ldots, 4$, and $\phi_{K}^{\prime}>0$ on $\mathbb{R}$, we get:

$$
\begin{aligned}
& \left|5 \mu_{p} \int_{\mathbb{R}}\left(\partial_{x} u\right)^{2} \phi_{K}^{\prime \prime \prime}(\bar{x})-\mu_{p} \int_{\mathbb{R}} u^{2} \phi_{K}^{(5)}(\bar{x})+\int_{\mathbb{R}} u^{2} \phi_{K}^{\prime \prime \prime}(\bar{x})\right| \\
& \lesssim \frac{1}{K^{2}} \int_{\mathbb{R}}\left(\left(\partial_{x} u\right)^{2}+u^{2}\right) \phi_{K}^{\prime}(\bar{x}) .
\end{aligned}
$$


Now, let us make the estimate of the nonlinear term: $\int_{\mathbb{R}} u^{p+2} \phi_{K}^{\prime}(\bar{x})$. Let $R_{0}>0$, we split the line in two regions:

$$
\begin{aligned}
\frac{2}{p+2} \int_{\mathbb{R}} u^{p+2} \phi_{K}^{\prime}(\bar{x}) & =\frac{2}{p+2} \int_{|x-\xi(t)|>R_{0}} u^{p+2} \phi_{K}^{\prime}(\bar{x})+\frac{2}{p+2} \int_{|x-\xi(t)|<R_{0}} u^{p+2} \phi_{K}^{\prime}(\bar{x}) \\
& =A+B .
\end{aligned}
$$

First, since $\| u\left((t, \cdot)-\varphi_{c_{0}, p, \mu_{p}}(\cdot-\xi(t)) \|_{C^{0}(\mathbb{R})} \leq \gamma_{0}\right.$ (by the Sobolev embedding of $H^{1}(\mathbb{R})$ into $C^{0}(\mathbb{R})$ ), we have $\sup _{x \in \mathbb{R}}|u(t, x)| \leq \sup _{x \in \mathbb{R}}\left|\varphi_{c_{0}, p, \mu_{p}}(x-\xi(t))\right|+\gamma_{0}$. Then it holds

$$
|A| \lesssim\left(e^{-\sqrt{c_{0}} R_{0}}+\gamma_{0}\right)^{p} \int_{\mathbb{R}} u^{2} \phi_{K}^{\prime}(\bar{x}) .
$$

Second, using that $\dot{\xi}(t) \geq 3 c_{0} / 4$, one can see that: $|\bar{x}| \geq-|x-\xi(t)|+\left|\left(\xi\left(t_{0}\right)-\xi(t)\right)-\frac{c_{0}}{2}\left(t_{0}-t\right)+x_{0}\right| \geq$ $-R_{0}+\frac{c_{0}}{4}\left(t_{0}-t\right)+x_{0}$. Then, using that $\phi_{K}^{\prime} \lesssim e^{-|\cdot| / K}$ on $\mathbb{R}$, and that $u(t)$ is uniformly bounded in $H^{1}(\mathbb{R})$, it holds

$$
|B| \lesssim e^{-x_{0} / K} e^{-c_{0}\left(t_{0}-t\right) / 4 K}
$$

Finally, combining (2.42)-2.46), taking $R_{0} \gg 1$ and $\gamma \ll 1$, and integrating $\frac{d}{d t} I_{x_{0}, t_{0}}(t)$ between $\left[t, t_{0}\right]$, we obtain

$$
I_{x_{0}, t_{0}}\left(t_{0}\right)+C_{0} \int_{t}^{t_{0}} \int_{\mathbb{R}}\left(\left(\partial_{x}^{2} u\right)^{2}+\left(\partial_{x} u\right)^{2}+u^{2}\right)(s, x) \phi_{K}^{\prime}(\bar{x}) d x d s \lesssim e^{-x_{0} / K}+I_{x_{0}, t_{0}}(t),
$$

where $C_{0}$ is a positive constant. This gives in particular (2.41).

We present now an immediate consequence of Step 1 and the exponential decay assumption on $u(t, x)$.

Step 2. For $x_{0}>0$ and $t_{0} \in \mathbb{R}$, we claim that:

$$
\int_{\mathbb{R}} u^{2}\left(t_{0}, x\right) \phi_{K}\left(x-\xi\left(t_{0}\right)-x_{0}\right) d x+C_{0} \int_{-\infty}^{t_{0}} \int_{\mathbb{R}}\left(\left(\partial_{x}^{2} u\right)^{2}+\left(\partial_{x} u\right)^{2}+u^{2}\right)(s, x) \phi_{K}^{\prime}(\bar{x}) d x d s \lesssim e^{-x_{0} / K} .
$$

Proof of (2.48). In view of Step 1, it suffices to prove that: $I_{x_{0}, t_{0}}(t) \rightarrow 0$ as $t \rightarrow-\infty$. Let $R_{1}>0$, we divide $\mathbb{R}$ in two parts: $\left\{x<R_{1}\right\}$ and $\left\{x>R_{1}\right\}$, and we proceed as follow:

$$
I_{x_{0}, t_{0}}(t)=\int_{x>R_{1}} u^{2}(t, x+\xi(t)) \phi_{K}(\bar{x}+\xi(t))+\int_{x<R_{1}} u^{2}(t, x+\xi(t)) \phi_{K}(\bar{x}+\xi(t))=A+B .
$$

First, using that $|u(t, x+\xi(t))| \leq e^{-\sigma|x|}$ and $\left\|\phi_{K}\right\|_{L^{\infty}(\mathbb{R})} \leq 1$, it holds:

$$
A \lesssim e^{-2 \sigma R_{1}} .
$$

Second, using that $\dot{\xi}(t) \geq 3 c_{0} / 4$, one can check that: $\bar{x}+\xi(t) \leq x-\frac{c_{0}}{4}\left(t_{0}-t\right)-x_{0}$. Then, since $\phi_{K}$ is increasing on $\mathbb{R}$, and the $L^{2}$-norm of $u(t, x)$ is conserved, it holds:

$$
B \lesssim \phi_{K}\left(R_{1}-\frac{c_{0}}{4}\left(t_{0}-t\right)-x_{0}\right)
$$

Letting $t \rightarrow-\infty$ in 2.51), and taking $R_{1}$ large, we obtain that: $\lim _{t \rightarrow-\infty} I_{x_{0}, t_{0}}(t)=0$, and claim 2.48 follows.

Next, we define the $H^{2}$-localized energy. Let $x_{0}>0$ and $t_{0} \in \mathbb{R}$. For all $t \leq t_{0}$, we define:

$$
J_{x_{0}, t_{0}}(t)=\int_{\mathbb{R}}\left(\frac{1}{2}\left(\partial_{x}^{2} u\right)^{2}+\frac{1}{2}\left(\partial_{x} u\right)^{2}-\frac{1}{(p+1)(p+2)} u^{p+2}\right)(t, x) \phi_{K}(\bar{x}) d x .
$$

As in Step 1, we establish the monotonicity property of the functional $J_{x_{0}, t_{0}}(t)$. 
Step 3. For $x_{0}>0$ and $t \leq t_{0}$, we claim that:

$$
J_{t_{0}, x_{0}}\left(t_{0}\right)-J_{x_{0}, t_{0}}(t) \lesssim e^{-x_{0} / K}
$$

Proof of 2.53). We just present the strategy since the proof is identical to 2.41). First, we differentiate $J_{x_{0}, t_{0}}(t)$ with respect to time, we apply the Virial Type Identity II (see Subsection 2.5), and we order the terms following the same logic as in 2.42). Observe that, we just need to estimate carefully the term: $\left|\frac{5 \mu_{p}}{2} \int_{\mathbb{R}}\left(\partial_{x}^{3} u\right)^{2} \phi_{K}^{\prime \prime \prime}(\bar{x})\right| \lesssim \frac{1}{K^{2}} \int_{\mathbb{R}}\left(\partial_{x}^{3} u\right)^{2} \phi_{K}^{\prime}(\bar{x})$. The rest of the term in Virial Identity II are bounded by: $\sum_{i=0}^{2} \int_{\mathbb{R}}\left(\partial_{x}^{j} u\right)^{2} \phi_{K}^{\prime}(\bar{x})$ (we use that $u(t)$ is $H^{2}$-bounded). Then, integration $\frac{d}{d t} J_{x_{0}, t_{0}}(t)$ between $\left[t, t_{0}\right]$, using (2.48), and choosing $K>0$ correctly, we get

$$
J_{x_{0}, t_{0}}\left(t_{0}\right)+C_{1} \int_{t}^{t_{0}} \int_{\mathbb{R}}\left(\left(\partial_{x}^{4} u\right)^{2}+\left(\partial_{x}^{3} u\right)^{2}+\left(\partial_{x}^{2} u\right)^{2}+\left(\partial_{x} u\right)^{2}+u^{2 p+2}\right)(s, x) \phi_{K}^{\prime}(\bar{x}) d x d s \lesssim e^{-x_{0} / K}+J_{x_{0}, t_{0}}(t),
$$

with $C_{1}>0$. This implies 2.53.

Estimate 2.54 and the decay assumption on $u(t, x)$ lead to the following control.

Step 4. For $x_{0}>0$ and $t_{0} \in \mathbb{R}$, we claim that:

$$
\begin{aligned}
& \int_{\mathbb{R}}\left(\left(\partial_{x}^{2} u\right)^{2}+\left(\partial_{x} u\right)^{2}\right)\left(t_{0}, x\right) \phi_{K}\left(x-\xi\left(t_{0}\right)-x_{0}\right) d x \\
& \quad+\int_{-\infty}^{t_{0}} \int_{\mathbb{R}}\left(\left(\partial_{x}^{4} u\right)^{2}+\left(\partial_{x}^{3} u\right)^{2}+\left(\partial_{x}^{2} u\right)^{2}+\left(\partial_{x} u\right)^{2}+u^{2 p+2}\right)(s, x) \phi_{K}^{\prime}(\bar{x}) d x d s \lesssim e^{-x_{0} / K} .
\end{aligned}
$$

Let us prove 2.55). In view of 2.54), it suffices to prove that $\lim _{t \rightarrow-\infty} J_{x_{0}, t_{0}}(t)=0$. Remark that $J_{x_{0}, t_{0}}(t) \lesssim \sum_{j=0}^{2} \int_{\mathbb{R}}\left(\partial_{x}^{j} u\right)^{2}(t, x) \phi_{K}(\bar{x})$ (since $u(t)$ is bounded in $\left.H^{1}(\mathbb{R})\right)$. From 2.48), we know that

$$
\sum_{k=0}^{2} \int_{-\infty}^{t_{0}} \int_{\mathbb{R}}\left(\partial_{x}^{k} u\right)^{2} \phi_{K}^{\prime}(\bar{x}) \lesssim e^{-x_{0} / K}
$$

Then

$$
\sum_{k=0}^{2} \int_{-\infty}^{t_{0}} \int_{x<\xi\left(t_{0}\right)-\frac{c_{0}}{2}\left(t_{0}-t\right)+x_{0}}\left(\partial_{x}^{k} u\right)^{2} e^{\left(x-\xi\left(t_{0}\right)+\frac{c_{0}}{2}\left(t_{0}-t\right)\right) / K} \lesssim 1,
$$

since $e^{\bar{x} / K} \lesssim \phi_{K}^{\prime}(\bar{x})$ for $\bar{x}<0$. Thus, passing to the limit as $x_{0}$ tends to $+\infty$ in 2.57) and multiplying by $e^{-x_{0} / K}$, we get

$$
\sum_{k=0}^{2} \int_{-\infty}^{t_{0}} \int_{\mathbb{R}}\left(\partial_{x}^{k} u\right)^{2} e^{\bar{x} / K} \lesssim e^{-x_{0} / K}
$$

and then, since $\phi(\bar{x}) \lesssim e^{\bar{x} / K}$ for all $x \in \mathbb{R}$,

$$
\sum_{k=0}^{2} \int_{-\infty}^{t_{0}} \int_{\mathbb{R}}\left(\partial_{x}^{k} u\right)^{2} \phi_{K}(\bar{x}) \lesssim e^{-x_{0} / K}
$$

Thus, we infer that

$$
\liminf _{t \rightarrow-\infty} \sum_{k=0}^{2} \int_{\mathbb{R}}\left(\partial_{x}^{k} u\right)^{2} \phi_{K}(\bar{x})=0
$$

We prove now that all derivatives of $u(t, x)$ are uniformly localized in $\mathbb{R}$.

Step 5. For $x_{0}>0, t_{0} \in \mathbb{R}$ and $k \in \mathbb{N}$, we claim that:

$$
\int_{\mathbb{R}}\left(\partial_{x}^{k} u\right)^{2}(t, x) \phi_{K}\left(x-\xi\left(t_{0}\right)-x_{0}\right) d x+\sum_{j=0}^{2} \int_{-\infty}^{t_{0}} \int_{\mathbb{R}}\left(\partial_{x}^{k+j} u\right)^{2}(s, x) \phi_{K}^{\prime}(\bar{x}) d x d s \lesssim e^{-x_{0} / K} .
$$


Proof of 2.61). We set: $I_{x_{0}, t_{0}}^{k}(t)=\int_{\mathbb{R}}\left(\partial_{x}^{k} u\right)^{2}(t, x) \phi_{K}(\bar{x})$ and we proceed by induction on $k$. First, from Step 2 and Step 4, the estimate (2.61) holds for $k=0,1,2$. We assume that (2.61) still true for $0 \leq j \leq k-1$. From the induction hypothesis one can deduce that $\int_{\mathbb{R}}\left(\partial_{x}^{k-1} u\right)^{2}\left(t_{0}, x+\xi\left(t_{0}\right) e^{|x| / K} \lesssim 1\right.$ (in particular $u(t)$ is bounded in $H^{k-1}(\mathbb{R})$, see Step 6 below). Also, $\int_{-\infty}^{t_{0}} \int_{\mathbb{R}}\left(\partial_{x}^{k} u\right)^{2} \phi_{K}(\bar{x}) \lesssim e^{-x_{0} / K}$, which implies that $\lim _{x \rightarrow-\infty} I_{x_{0}, t_{0}}^{k}(t)=0$. Now, by computing the time variation of $I_{x_{0}, t_{0}}^{(k)}(t)$, with the aid of Virial Type Identity I applied with $\partial_{x}^{k} u(t, x)$, we get:

$$
\begin{aligned}
\frac{d}{d t} I_{x_{0}, t_{0}}^{k}(t)=- & 5 \int_{\mathbb{R}}\left(\partial_{x}^{k+2} u\right)^{2} \phi_{K}^{\prime}(\bar{x})-3 \int_{\mathbb{R}}\left(\partial_{x}^{k+1} u\right)^{2} \phi_{K}^{\prime}(\bar{x})-\frac{c_{0}}{2} \int_{\mathbb{R}}\left(\partial_{x}^{k} u\right)^{2} \phi_{K}^{\prime}(\bar{x}) \\
+ & 5 \mu_{p} \int_{\mathbb{R}}\left(\partial_{x}^{k+1} u\right)^{2} \phi_{K}^{\prime \prime \prime}(\bar{x})-\mu_{p} \int_{\mathbb{R}}\left(\partial_{x}^{k} u\right)^{2} \phi_{K}^{(5)}(\bar{x})+\int_{\mathbb{R}}\left(\partial_{x}^{k} u\right)^{2} \phi_{K}^{\prime \prime \prime}(\bar{x}) \\
& -\frac{2}{p+1} \int_{\mathbb{R}}\left(\partial_{x}^{k} u\right)\left(\partial_{x}^{k+1} u^{p+1}\right) \phi_{K}(\bar{x}) .
\end{aligned}
$$

The terms in the second line of 2.62 are clearly bounded by: $\int_{\mathbb{R}}\left(\left(\partial_{x}^{k+1} u\right)^{2}+\left(\partial_{x}^{k} u\right)^{2}\right) \phi_{K}^{\prime}(\bar{x})$. Let us estimate the nonlinear term: $N=-\frac{2}{p+1} \int_{\mathbb{R}}\left(\partial_{x}^{k} u\right)\left(\partial_{x}^{k+1} u^{p+1}\right) \phi_{K}(\bar{x})$. By differentiating thrice $u^{p+1}$, we get

$$
\begin{aligned}
N=-2 \int_{\mathbb{R}} \partial_{x}^{k-2}\left[\left(\partial_{x}^{3} u\right) u^{p}\right]\left(\partial_{x}^{k} u\right) \phi_{K}(\bar{x})-6 p \int_{\mathbb{R}} \partial_{x}^{k-2}\left[\left(\partial_{x}^{2} u\right)\left(\partial_{x} u\right) u^{p-1}\right]\left(\partial_{x}^{k} u\right) \phi_{K}(\bar{x}) \\
\quad-2(p-1) \int_{\mathbb{R}} \partial_{x}^{k-2}\left[\left(\partial_{x} u\right)^{3} u^{p-2}\right]\left(\partial_{x}^{k} u\right) \phi_{K}(\bar{x}) \\
=N_{1}+N_{2}+N_{3} .
\end{aligned}
$$

We claim that:

$$
N \lesssim \sum_{j=1}^{k+1} \int_{\mathbb{R}}\left(\partial_{x}^{j} u\right)^{2} \phi_{K}(\bar{x}) .
$$

Let us estimate $N_{1}$. By applying the Leibniz formula on $\partial_{x}^{k-2}\left[\left(\partial_{x}^{3} u\right) u^{p}\right]$, we can rewrite $N_{1}$ as

$$
N_{1}=-2 \sum_{j=0}^{k-2} C_{k-2}^{j} \int_{\mathbb{R}}\left(\partial_{x}^{k-j+1} u\right)\left(\partial_{x}^{k} u\right)\left(\partial_{x}^{j} u^{p}\right) \phi_{K}(\bar{x}) .
$$

Now, using that $\left\|\partial_{x}^{j} u^{p}\right\|_{L^{\infty}(\mathbb{R})} \lesssim 1$ for $0 \leq j \leq k-2$, and applying the Young's inequality, we infer that

$$
N_{1} \lesssim \sum_{j=3}^{k+1} \int_{\mathbb{R}}\left(\partial_{x}^{j} u\right)^{2} \phi_{K}(\bar{x})
$$

The estimates of $N_{2}$ and $N_{3}$ follow by applying the same arguments. When estimating $N_{2}$, you just have to pay attention to two terms: $\int_{\mathbb{R}}\left(\partial_{x}^{2} u\right)^{2}\left(\partial_{x}^{k} u\right)\left(\partial_{x}^{k-3} u^{p-1}\right) \phi_{K}(\bar{x})$ and $\int_{\mathbb{R}}\left(\partial_{x}^{2} u\right)\left(\partial_{x}^{k-1} u\right)\left(\partial_{x}^{k} u\right) u^{p-1} \phi_{K}(\bar{x})$, which require one integration by part.

Finally, by integrating $I_{x_{0}, t_{0}}^{k}(t)$ between $\left[-\infty, t_{0}\right]$, and using that $\sum_{j=0}^{k+1} \int_{-\infty}^{t_{0}} \int_{\mathbb{R}}\left(\partial_{x}^{j} u\right)^{2} \phi_{K}(\bar{x}) \lesssim e^{-x_{0} / K}$ and $I_{x_{0}, t_{0}}^{k}(-\infty)=0$, we get 2.61).

Step 6. For all $k \in \mathbb{N}$, we claim that:

$$
\int_{\mathbb{R}}\left[\partial_{x}^{k} u\left(t_{0}, x+\xi\left(t_{0}\right)\right)\right]^{2}(t, x) e^{|x| / K} \lesssim 1 .
$$

Proof of (2.67). From Step 5, we know that:

$$
\int_{\mathbb{R}}\left[\partial_{x}^{k} u\left(t_{0}, x+\xi\left(t_{0}\right)\right)\right]^{2} \phi_{K}\left(x-x_{0}\right) \lesssim e^{-x_{0} / K},
$$


and then

$$
\int_{x<x_{0}}\left[\partial_{x}^{k} u\left(t_{0}, x+\xi\left(t_{0}\right)\right)\right]^{2} e^{x / K} \lesssim 1
$$

since $e^{\left(x-x_{0}\right) / K} \lesssim \phi_{K}\left(x-x_{0}\right)$ for $x<x_{0}$. Now, passing to the limit as $x_{0}$ tends to $+\infty$ in 2.69 , we get the exponential decay property by the right:

$$
\int_{\mathbb{R}}\left[\partial_{x}^{k} u\left(t_{0}, x+\xi\left(t_{0}\right)\right)\right]^{2} e^{x / K} \lesssim 1
$$

For the left exponential decay property, one can remark that the equation of gKW is invariant by the transformation $u(t, x) \mapsto u(-t,-x)$, moreover $u(-t,-x)$ is uniformly localized. Then, repeating the same analysis with $u(-t,-x)$, we get the claim (2.67). In particular, applying the Gagliardo-Nirenberg inequality, we infer that for all $k \in \mathbb{N}$,

$$
\begin{aligned}
\left\|\left[\partial_{x}^{k} u\left(t_{0}, \cdot+\xi\left(t_{0}\right)\right)\right] e^{|\cdot| / 2 K}\right\|_{L^{\infty}(\mathbb{R})} & \leq C_{G}\left\|\left[\partial_{x}^{k} u\left(t_{0}, \cdot+\xi\left(t_{0}\right)\right)\right] e^{|\cdot| / 2 K}\right\|_{L^{2}(\mathbb{R})}^{1 / 2} \times \\
& \left(\left\|\left[\partial_{x}^{k} u\left(t_{0}, \cdot+\xi\left(t_{0}\right)\right)\right] e^{|\cdot| / 2 K}\right\|_{L^{2}(\mathbb{R})}^{2}+\left\|\left[\partial_{x}^{k+1} u\left(t_{0}, \cdot+\xi\left(t_{0}\right)\right)\right] e^{|\cdot| / 2 K}\right\|_{L^{2}(\mathbb{R})}^{2}\right)^{1 / 4} \lesssim 1,
\end{aligned}
$$

and this prove 2.14 .

\subsection{Time variation of the localized mass}

In this section, we compute the time variation of $L^{2}$-localized norm.

Lemma 2.5 (Virial Type Identity I). Assume that $\mu_{0}>0$ and $p \in \mathbb{N}^{*}$. Let $u(t) \in H^{2}(\mathbb{R})$ be a solution of $\mu_{0}-g K W$ equation. Then, for any smooth $\left(C^{5}\right)$ space function $g: \mathbb{R} \rightarrow \mathbb{R}$, it holds

$$
\begin{aligned}
\frac{d}{d t} \int_{\mathbb{R}} u^{2} g= & -5 \mu_{0} \int_{\mathbb{R}}\left(\partial_{x}^{2} u\right)^{2} g^{\prime}-3 \int_{\mathbb{R}}\left(\partial_{x} u\right)^{2} g^{\prime}+\int_{\mathbb{R}} u^{2} g^{\prime \prime \prime} \\
& +5 \mu_{0} \int_{\mathbb{R}}\left(\partial_{x} u\right)^{2} g^{\prime \prime \prime}-\mu_{0} \int_{\mathbb{R}} u^{2} g^{(5)}+\frac{2}{p+2} \int_{\mathbb{R}} u^{p+2} g^{\prime} .
\end{aligned}
$$

Proof. We have

$$
\frac{d}{d t} \int_{\mathbb{R}} u^{2} g=2 \int_{\mathbb{R}}\left(\partial_{t} u\right) u g
$$

and using that $u(t, x)$ is a solution of $\mu_{0}$-gKW equation, we obtain

$$
\begin{aligned}
\frac{d}{d t} \int_{\mathbb{R}} u^{2} g & =-\frac{2}{p+1} \int_{\mathbb{R}}\left(\partial_{x} u^{p+1}\right) u g-2 \int_{\mathbb{R}}\left(\partial_{x}^{3} u\right) u g+2 \mu_{0} \int_{\mathbb{R}}\left(\partial_{x}^{5} u\right) u g \\
& =I+J+K .
\end{aligned}
$$

Let us compute $I$ :

$$
\begin{aligned}
I & =\frac{2}{p+1} \int_{\mathbb{R}} u^{p+1} \partial_{x}(u g) \\
& =\frac{2}{(p+1)(p+2)} \int_{\mathbb{R}}\left(\partial_{x} u^{p+2}\right) g+\frac{2}{p+1} \int_{\mathbb{R}} u^{p+2} g^{\prime} \\
& =\frac{2}{p+2} \int_{\mathbb{R}} u^{p+2} g^{\prime} .
\end{aligned}
$$


Let us compute $J$ :

$$
\begin{aligned}
J & =2 \int_{\mathbb{R}}\left(\partial_{x}^{2} u\right) \partial_{x}(u g) \\
& =\int_{\mathbb{R}} \partial_{x}\left[\left(\partial_{x} u\right)^{2}\right] g+2 \int_{\mathbb{R}}\left(\partial_{x}^{2} u\right) u g^{\prime} \\
& =-\int_{\mathbb{R}}\left(\partial_{x} u\right)^{2} g^{\prime}+2 \int_{\mathbb{R}}\left(\partial_{x}^{2} u\right) u g^{\prime}
\end{aligned}
$$

with

$$
\begin{aligned}
2 \int_{\mathbb{R}}\left(\partial_{x}^{2} u\right) u g^{\prime} & =-2 \int_{\mathbb{R}}\left(\partial_{x} u\right) \partial_{x}\left(u g^{\prime}\right) \\
& =-2 \int_{\mathbb{R}}\left(\partial_{x} u\right)^{2} g^{\prime}-\int_{\mathbb{R}}\left(\partial_{x} u^{2}\right) g^{\prime \prime} \\
& =-2 \int_{\mathbb{R}}\left(\partial_{x} u\right)^{2}+\int_{\mathbb{R}} u^{2} g^{\prime \prime \prime}
\end{aligned}
$$

and then summing 2.76 and 2.77, we get

$$
J=-3 \int_{\mathbb{R}}\left(\partial_{x} u\right)^{2} g^{\prime}+\int_{\mathbb{R}} u^{2} g^{\prime \prime \prime} .
$$

Let us compute $K$ :

$$
\begin{aligned}
K & =-2 \mu_{0} \int_{\mathbb{R}}\left(\partial_{x}^{4} u\right) \partial_{x}(u g) \\
& =-2 \mu_{0} \int_{\mathbb{R}}\left(\partial_{x}^{4} u\right)\left(\partial_{x} u\right) g-2 \mu_{0} \int_{\mathbb{R}}\left(\partial_{x}^{4} u\right) u g^{\prime}=K_{1}+K_{2}
\end{aligned}
$$

Let us tackle $K_{1}$ :

$$
\begin{aligned}
K_{1} & =2 \mu_{0} \int_{\mathbb{R}}\left(\partial_{x}^{3} u\right) \partial_{x}\left[\left(\partial_{x} u\right) g\right] \\
& =\mu_{0} \int_{\mathbb{R}} \partial_{x}\left[\left(\partial_{x}^{2} u\right)^{2}\right] g+2 \mu_{0} \int_{\mathbb{R}}\left(\partial_{x}^{3} u\right)\left(\partial_{x} u\right) g^{\prime} \\
& =-\mu_{0} \int_{\mathbb{R}}\left(\partial_{x}^{2} u\right)^{2} g^{\prime}+K_{11}
\end{aligned}
$$

with

$$
\begin{aligned}
K_{11} & =-2 \mu_{0} \int_{\mathbb{R}}\left(\partial_{x}^{2} u\right)\left[\left(\partial_{x}^{2} u\right) g^{\prime}+\left(\partial_{x} u\right) g^{\prime \prime}\right] \\
& =-2 \mu_{0} \int_{\mathbb{R}}\left(\partial_{x}^{2} u\right)^{2} g^{\prime}-\mu_{0} \int_{\mathbb{R}} \partial_{x}\left[\left(\partial_{x} u\right)^{2}\right] g^{\prime \prime} \\
& =-2 \mu_{0} \int_{\mathbb{R}}\left(\partial_{x}^{2} u\right)^{2} g^{\prime}+\mu_{0} \int_{\mathbb{R}}\left(\partial_{x} u\right)^{2} g^{\prime \prime \prime},
\end{aligned}
$$

and then summing 2.82 and 2.81, we get

$$
K_{1}=-3 \mu_{0} \int_{\mathbb{R}}\left(\partial_{x}^{2} u\right)^{2} g^{\prime}+\mu_{0} \int_{\mathbb{R}}\left(\partial_{x} u\right)^{2} g^{\prime \prime \prime} .
$$


Let us tackle $K_{2}$ :

$$
\begin{aligned}
K_{2} & =2 \mu_{0} \int_{\mathbb{R}}\left(\partial_{x}^{3} u\right) \partial_{x}\left(u g^{\prime}\right) \\
& =2 \mu_{0} \int_{\mathbb{R}}\left(\partial_{x}^{3} u\right)\left(\partial_{x} u\right) g^{\prime}+2 \mu_{0} \int_{\mathbb{R}}\left(\partial_{x}^{3} u\right) u g^{\prime \prime}=K_{21}+K_{22},
\end{aligned}
$$

with

$$
\begin{aligned}
K_{21} & =-2 \mu_{0} \int_{\mathbb{R}}\left(\partial_{x}^{2} u\right) \partial_{x}\left[\left(\partial_{x} u\right) g^{\prime}\right] \\
& =-2 \mu_{0} \int_{\mathbb{R}}\left(\partial_{x}^{2} u\right)^{2} g^{\prime}-\mu_{0} \int_{\mathbb{R}} \partial_{x}\left[\left(\partial_{x} u\right)^{2}\right] g^{\prime \prime} \\
& =-2 \mu_{0} \int_{\mathbb{R}}\left(\partial_{x}^{2} u\right)^{2} g^{\prime}+\mu_{0} \int_{\mathbb{R}}\left(\partial_{x} u\right)^{2} g^{\prime \prime \prime},
\end{aligned}
$$

and

$$
\begin{aligned}
K_{22} & =-2 \mu_{0} \int_{\mathbb{R}}\left(\partial_{x}^{2} u\right) \partial_{x}\left(u g^{\prime \prime}\right) \\
& =-\mu_{0} \int_{\mathbb{R}} \partial_{x}\left[\left(\partial_{x} u\right)^{2}\right] g^{\prime \prime}+2 \mu_{0} \int_{\mathbb{R}}\left(\partial_{x} u\right) \partial_{x}\left(u g^{\prime \prime \prime}\right) \\
& =3 \mu_{0} \int_{\mathbb{R}}\left(\partial_{x} u\right)^{2} g^{\prime \prime \prime}+\mu_{0} \int_{\mathbb{R}} \partial_{x}\left(u^{2}\right) g^{(4)} \\
& =3 \mu_{0} \int_{\mathbb{R}}\left(\partial_{x} u\right)^{2} g^{\prime \prime \prime}-\mu_{0} \int_{\mathbb{R}} u^{2} g^{(5)} .
\end{aligned}
$$

Then, summing (2.85) and 2.84), we get

$$
K_{2}=-2 \mu_{0} \int_{\mathbb{R}}\left(\partial_{x}^{2} u\right)^{2} g^{\prime}+4 \mu_{0} \int_{\mathbb{R}}\left(\partial_{x} u\right)^{2} g^{\prime \prime \prime}-\mu_{0} \int_{\mathbb{R}} u^{2} g^{(5)},
$$

and summing $(2.82)$ and 2.86$)$, we find

$$
K=-5 \mu_{0} \int_{\mathbb{R}}\left(\partial_{x}^{2} u\right)^{2} g^{\prime}+5 \mu_{0} \int_{\mathbb{R}}\left(\partial_{x} u\right)^{2} g^{\prime \prime \prime}-\mu_{0} \int_{\mathbb{R}} u^{2} g^{(5)} .
$$

Finally, by adding (2.75), 2.78) and (2.87), we obtain (2.72), and this prove the lemma.

\subsection{Time variation of the localized energy}

In this section, we compute the time variation of $H^{2}$-localized norm.

Lemma 2.6 (Virial Type Identity II). Assume that $\mu_{0}>0$ and $p \in \mathbb{N}^{*}$. Let $u(t) \in H^{4}(\mathbb{R})$ be a solution of $\mu_{0}-g K W$ equation. Then, for any smooth $\left(C^{5}\right)$ space function: $g: \mathbb{R} \rightarrow \mathbb{R}$, it holds

$$
\begin{aligned}
& \frac{d}{d t} \int_{\mathbb{R}}\left(\frac{\mu_{0}}{2}\left(\partial_{x}^{2} u\right)^{2}+\frac{1}{2}\left(\partial_{x} u\right)^{2}-\frac{1}{(p+1)(p+2)} u^{p+2}\right) g \\
& =-\frac{5 \mu_{0}^{2}}{2} \int_{\mathbb{R}}\left(\partial_{x}^{4} u\right)^{2} g^{\prime}-4 \mu_{0} \int_{\mathbb{R}}\left(\partial_{x}^{3} u\right)^{2} g^{\prime}-\frac{3}{2} \int_{\mathbb{R}}\left(\partial_{x}^{2} u\right)^{2} g^{\prime}-\frac{1}{2(p+1)^{2}} \int_{\mathbb{R}} u^{2 p+2} g^{\prime} \\
& \quad+\frac{5 \mu_{0}^{2}}{2} \int_{\mathbb{R}}\left(\partial_{x}^{3} u\right)^{2} g^{\prime \prime \prime}-\frac{\mu_{0}^{2}}{2} \int_{\mathbb{R}}\left(\partial_{x}^{2} u\right)^{2} g^{(5)}+3 \mu_{0} \int_{\mathbb{R}}\left(\partial_{x}^{2} u\right)^{2} g^{\prime \prime \prime}-\frac{\mu_{0}}{2} \int_{\mathbb{R}}\left(\partial_{x} u\right)^{2} g^{(5)}+\frac{1}{2} \int_{\mathbb{R}}\left(\partial_{x} u\right)^{2} g^{\prime \prime \prime} \\
& \quad+3 \mu_{0} \int_{\mathbb{R}}\left(\partial_{x}^{2} u\right)^{2} u^{p} g^{\prime}-\mu_{0} p(p-1) \int_{\mathbb{R}}\left(\partial_{x} u\right)^{4} u^{p-2} g^{\prime}-\frac{15 \mu_{0} p}{6} \int_{\mathbb{R}}\left(\partial_{x} u\right)^{3} u^{p-1} g^{\prime \prime}+2 \int_{\mathbb{R}}\left(\partial_{x} u\right)^{2} u^{p} g^{\prime} \\
& \quad-\frac{5 \mu_{0}}{2} \int_{\mathbb{R}}\left(\partial_{x} u\right)^{2} u^{p} g^{\prime \prime \prime}-\frac{1}{(p+1)(p+2)} \int_{\mathbb{R}} u^{p+2} g^{\prime \prime \prime}+\frac{\mu_{0}}{(p+1)(p+2)} \int_{\mathbb{R}} u^{p+2} g^{(5)} .
\end{aligned}
$$


Proof. We set:

$$
I=\frac{\mu_{0}}{2} \frac{d}{d t} \int_{\mathbb{R}}\left(\partial_{x}^{2} u\right)^{2} g, \quad J=\frac{1}{2} \frac{d}{d t} \int_{\mathbb{R}}\left(\partial_{x} u\right)^{2} g, \quad \text { and } \quad K=-\frac{1}{(p+1)(p+2)} \frac{d}{d t} \int_{\mathbb{R}} u^{p+2} g .
$$

Let us start with $K$ :

$$
K=-\frac{1}{p+1} \int_{\mathbb{R}} u^{p+1}\left(\partial_{t} u\right) g,
$$

using that $u(t, x)$ satisfies the $\mu_{0}$-gKW equation, we get

$$
\begin{aligned}
K & =\frac{1}{p+1} \int_{\mathbb{R}}\left(\partial_{x} u\right) u^{2 p+1} g+\frac{1}{p+1} \int_{\mathbb{R}}\left(\partial_{x}^{3} u\right) u^{p+1} g-\frac{\mu_{0}}{p+1} \int_{\mathbb{R}}\left(\partial_{x}^{5} u\right) u^{p+1} g \\
& =-\frac{1}{2(p+1)^{2}} \int_{\mathbb{R}} u^{2 p+2} g^{\prime}+K_{1}+K_{2} .
\end{aligned}
$$

Let us compute $K_{1}$ :

$$
\begin{aligned}
K_{1} & =-\frac{1}{p+1} \int_{\mathbb{R}}\left(\partial_{x}^{2} u\right) \partial_{x}\left(u^{p+1} g\right) \\
& =-\int_{\mathbb{R}}\left(\partial_{x}^{2} u\right)\left(\partial_{x} u\right) u^{p} g-\frac{1}{p+1} \int_{\mathbb{R}}\left(\partial_{x}^{2} u\right) u^{p+1} g^{\prime}=K_{11}+K_{12},
\end{aligned}
$$

with

$$
K_{11}=-\frac{1}{2} \int_{\mathbb{R}} \partial_{x}\left[\left(\partial_{x} u\right)^{2}\right] u^{p} g=\frac{p}{2} \int_{\mathbb{R}}\left(\partial_{x} u\right)^{3} u^{p-1} g+\frac{1}{2} \int_{\mathbb{R}}\left(\partial_{x} u\right)^{2} u^{p} g^{\prime},
$$

and

$$
\begin{aligned}
K_{12} & =\frac{1}{p+1} \int_{\mathbb{R}}\left(\partial_{x} u\right) \partial_{x}\left(u^{p+1} g^{\prime}\right) \\
& =\int_{\mathbb{R}}\left(\partial_{x} u\right)^{2} u^{p} g^{\prime}+\frac{1}{(p+1)(p+2)} \int_{\mathbb{R}} \partial_{x}\left(u^{p+2}\right) g^{\prime \prime} \\
& =\int_{\mathbb{R}}\left(\partial_{x} u\right)^{2} u^{p} g^{\prime}-\frac{1}{(p+1)(p+2)} \int_{\mathbb{R}} u^{p+2} g^{\prime \prime \prime},
\end{aligned}
$$

and summing (2.94) and 2.93), we get

$$
K_{1}=\frac{p}{2} \int_{\mathbb{R}}\left(\partial_{x} u\right)^{3} u^{p+1} g+\frac{3}{2} \int_{\mathbb{R}}\left(\partial_{x} u\right)^{2} u^{p} g^{\prime}-\frac{1}{(p+1)(p+2)} \int_{\mathbb{R}} u^{p+2} g^{\prime \prime \prime} .
$$

Now, let us compute $K_{2}$ :

$$
\begin{aligned}
K_{2} & =\frac{\mu_{0}}{p+1} \int_{\mathbb{R}}\left(\partial_{x}^{4} u\right) \partial_{x}\left(u^{p+1} g\right) \\
& =\mu_{0} \int_{\mathbb{R}}\left(\partial_{x}^{4} u\right)\left(\partial_{x} u\right) u^{p} g+\frac{\mu_{0}}{p+1} \int_{\mathbb{R}}\left(\partial_{x}^{4} u\right) u^{p+1} g^{\prime}=K_{21}+K_{22} .
\end{aligned}
$$

$K_{21}$ give us

$$
\begin{aligned}
K_{21} & =-\mu_{0} \int_{\mathbb{R}}\left(\partial_{x}^{3} u\right) \partial_{x}\left[\left(\partial_{x} u\right) u^{p} g\right] \\
& =-\mu_{0} \int_{\mathbb{R}}\left(\partial_{x}^{3} u\right)\left(\partial_{x}^{2} u\right) u^{p} g-\mu_{0} p \int_{\mathbb{R}}\left(\partial_{x}^{3} u\right)\left(\partial_{x} u\right)^{2} u^{p-1} g-\mu_{0} \int_{\mathbb{R}}\left(\partial_{x}^{3} u\right)\left(\partial_{x} u\right) u^{p} g^{\prime} \\
& =-\mu_{0} \int_{\mathbb{R}}\left(\partial_{x}^{3} u\right)\left(\partial_{x}^{2} u\right) u^{p} g+K_{211}+K_{212},
\end{aligned}
$$


with

$$
\begin{aligned}
& -\mu_{0} \int_{\mathbb{R}}\left(\partial_{x}^{3} u\right)\left(\partial_{x}^{2} u\right) u^{p} g \\
& \quad=-\frac{\mu_{0}}{2} \int_{\mathbb{R}} \partial_{x}\left[\left(\partial_{x}^{2} u\right)^{2}\right] u^{p} g \\
& \quad=\frac{\mu_{0} p}{2} \int_{\mathbb{R}}\left(\partial_{x}^{2} u\right)^{2}\left(\partial_{x} u\right) u^{p-1} g+\frac{\mu_{0}}{2} \int_{\mathbb{R}}\left(\partial_{x}^{2} u\right)^{2} u^{p} g^{\prime},
\end{aligned}
$$

and

$$
\begin{aligned}
K_{211}= & \mu_{0} p \int_{\mathbb{R}}\left(\partial_{x}^{2} u\right) \partial_{x}\left[\left(\partial_{x} u\right)^{2} u^{p-1} g\right] \\
= & 2 \mu_{0} p \int_{\mathbb{R}}\left(\partial_{x}^{2} u\right)^{2}\left(\partial_{x} u\right) u^{p-1} g+\mu_{0} p(p-1) \int_{\mathbb{R}}\left(\partial_{x}^{2} u\right)\left(\partial_{x} u\right)^{3} u^{p-2} g \\
& +\mu_{0} p \int_{\mathbb{R}}\left(\partial_{x}^{2} u\right)\left(\partial_{x} u\right)^{2} u^{p-1} g^{\prime},
\end{aligned}
$$

with

$$
\begin{aligned}
\mu_{0} p(p- & 1) \int_{\mathbb{R}}\left(\partial_{x}^{2} u\right)\left(\partial_{x} u\right)^{3} u^{p-2} g \\
& =\frac{\mu_{0} p(p-1)}{4} \int_{\mathbb{R}} \partial_{x}\left[\left(\partial_{x} u\right)^{4}\right] u^{p-2} g \\
& =-\frac{\mu_{0} p(p-1)(p-2)}{4} \int_{\mathbb{R}}\left(\partial_{x} u\right)^{5} u^{p-3} g-\frac{\mu_{0} p(p-1)}{4} \int_{\mathbb{R}}\left(\partial_{x} u\right)^{4} u^{p-2} g^{\prime},
\end{aligned}
$$

and

$$
\begin{aligned}
\mu_{0} p \int_{\mathbb{R}} & \left(\partial_{x}^{2} u\right)\left(\partial_{x} u\right)^{2} u^{p-1} g^{\prime} \\
& =\frac{\mu_{0} p}{3} \int_{\mathbb{R}} \partial_{x}\left[\left(\partial_{x} u\right)^{3}\right] u^{p-1} g^{\prime} \\
& =-\frac{\mu_{0} p(p-1)}{3} \int_{\mathbb{R}}\left(\partial_{x} u\right)^{4} u^{p-2} g^{\prime}-\frac{\mu_{0} p}{3} \int_{\mathbb{R}}\left(\partial_{x} u\right)^{3} u^{p-1} g^{\prime \prime} .
\end{aligned}
$$

Then summing 2.99), 2.100 and 2.101, we get

$$
\begin{aligned}
K_{211}= & 2 \mu_{0} p \int_{\mathbb{R}}\left(\partial_{x}^{2} u\right)^{2}\left(\partial_{x} u\right) u^{p-1} g-\frac{\mu_{0} p(p-1)(p-2)}{4} \int_{\mathbb{R}}\left(\partial_{x} u\right)^{5} u^{p-3} g \\
& -\frac{7 \mu_{0} p(p-1)}{12} \int_{\mathbb{R}}\left(\partial_{x} u\right)^{4} u^{p-2} g^{\prime}-\frac{\mu_{0} p}{3} \int_{\mathbb{R}}\left(\partial_{x} u\right)^{3} u^{p-2} g^{\prime \prime} .
\end{aligned}
$$

$K_{212}$ give us

$$
\begin{aligned}
K_{212} & =\mu_{0} \int_{\mathbb{R}}\left(\partial_{x}^{2} u\right) \partial_{x}\left[\left(\partial_{x} u\right) u^{p} g^{\prime}\right] \\
& =\mu_{0} \int_{\mathbb{R}}\left(\partial_{x}^{2} u\right)^{2} u^{p} g^{\prime}+\mu_{0} p \int_{\mathbb{R}}\left(\partial_{x}^{2} u\right)\left(\partial_{x} u\right)^{2} u^{p-1} g^{\prime}+\mu_{0} \int_{\mathbb{R}}\left(\partial_{x}^{2} u\right)\left(\partial_{x} u\right) u^{p} g^{\prime \prime},
\end{aligned}
$$


with

$$
\begin{aligned}
\mu_{0} p \int_{\mathbb{R}} & \left(\partial_{x}^{2} u\right)\left(\partial_{x} u\right)^{2} u^{p-1} g^{\prime} \\
& =\frac{\mu_{0} p}{3} \int_{\mathbb{R}} \partial_{x}\left[\left(\partial_{x} u\right)^{3}\right] u^{p-1} g^{\prime} \\
& =-\frac{\mu_{0} p(p-1)}{3} \int_{\mathbb{R}}\left(\partial_{x} u\right)^{4} u^{p-2} g^{\prime}-\frac{\mu_{0} p}{3} \int_{\mathbb{R}}\left(\partial_{x} u\right)^{3} u^{p-1} g^{\prime \prime},
\end{aligned}
$$

and

$$
\begin{aligned}
\mu_{0} \int_{\mathbb{R}}\left(\partial_{x}^{2} u\right)\left(\partial_{x} u\right) u^{p} g^{\prime \prime} \\
\quad=\frac{\mu_{0}}{2} \int_{\mathbb{R}} \partial_{x}\left[\left(\partial_{x} u\right)^{2}\right] u^{p} g^{\prime \prime} \\
\quad=-\frac{\mu_{0} p}{2} \int_{\mathbb{R}}\left(\partial_{x} u\right)^{3} u^{p-1} g^{\prime \prime}-\frac{\mu_{0}}{2} \int_{\mathbb{R}}\left(\partial_{x} u\right)^{2} u^{p} g^{\prime \prime \prime} .
\end{aligned}
$$

Then, summing 2.103), 2.104) and 2.105), we get

$$
\begin{aligned}
K_{212}= & \mu_{0} \int_{\mathbb{R}}\left(\partial_{x}^{2} u\right)^{2} u^{p} g^{\prime}-\frac{\mu_{0} p(p-1)}{3} \int_{\mathbb{R}}\left(\partial_{x} u\right)^{4} u^{p-2} g^{\prime} \\
& -\frac{5 \mu_{0} p}{6} \int_{\mathbb{R}}\left(\partial_{x} u\right)^{3} u^{p-1} g^{\prime \prime}-\frac{\mu_{0}}{2} \int_{\mathbb{R}}\left(\partial_{x} u\right)^{2} u^{p} g^{\prime \prime \prime},
\end{aligned}
$$

and summing 2.98, 2.102 and 2.106, we obtain

$$
\begin{aligned}
K_{21}= & \frac{5 \mu_{0} p}{2} \int_{\mathbb{R}}\left(\partial_{x}^{2} u\right)^{2}\left(\partial_{x} u\right) u^{p-1} g-\frac{\mu_{0} p(p-1)(p-2)}{4} \int_{\mathbb{R}}\left(\partial_{x} u\right)^{5} u^{p-3} g+\frac{3 \mu_{0}}{2} \int_{\mathbb{R}}\left(\partial_{x}^{2} u\right) u^{p} g^{\prime} \\
& -\frac{11 \mu_{0} p(p-1)}{12} \int_{\mathbb{R}}\left(\partial_{x} u\right)^{4} u^{p-2} g^{\prime}-\frac{7 \mu_{0} p}{6} \int_{\mathbb{R}}\left(\partial_{x} u\right)^{3} u^{p-1} g^{\prime \prime}-\frac{\mu_{0}}{2} \int_{\mathbb{R}}\left(\partial_{x} u\right)^{2} u^{p} g^{\prime \prime \prime} .
\end{aligned}
$$

Let us compute $K_{22}$ :

$$
\begin{aligned}
K_{22} & =-\frac{\mu_{0}}{p+1} \int_{\mathbb{R}}\left(\partial_{x}^{3} u\right) \partial_{x}\left(u^{p+1} g^{\prime}\right) \\
& =-\mu_{0} \int_{\mathbb{R}}\left(\partial_{x}^{3} u\right)\left(\partial_{x} u\right) u^{p} g^{\prime}-\frac{\mu_{0}}{p+1} \int_{\mathbb{R}}\left(\partial_{x}^{3} u\right) u^{p+1} g^{\prime \prime}=K_{221}+K_{222} .
\end{aligned}
$$

We check that

$$
\begin{aligned}
K_{221} & =\mu_{0} \int_{\mathbb{R}}\left(\partial_{x}^{2} u\right) \partial_{x}\left[\left(\partial_{x} u\right) u^{p} g^{\prime}\right] \\
& =\mu_{0} \int_{\mathbb{R}}\left(\partial_{x}^{2} u\right)^{2} u^{p} g^{\prime}+\mu_{0} p \int_{\mathbb{R}}\left(\partial_{x}^{2} u\right)\left(\partial_{x} u\right)^{2} u^{p-1} g^{\prime}+\mu_{0} \int_{\mathbb{R}}\left(\partial_{x}^{2} u\right)\left(\partial_{x} u\right) u^{p} g^{\prime \prime},
\end{aligned}
$$

with

$$
\begin{aligned}
\mu_{0} p \int_{\mathbb{R}} & \left(\partial_{x}^{2} u\right)\left(\partial_{x} u\right)^{2} u^{p-1} g^{\prime} \\
\quad & =\frac{\mu_{0} p}{3} \int_{\mathbb{R}} \partial_{x}\left[\left(\partial_{x} u\right)^{3}\right] u^{p-1} g^{\prime} \\
& =-\frac{\mu_{0} p(p-1)}{3} \int_{\mathbb{R}}\left(\partial_{x} u\right)^{4} u^{p-2} g^{\prime}-\frac{\mu_{0} p}{3} \int_{\mathbb{R}}\left(\partial_{x} u\right)^{3} u^{p-1} g^{\prime \prime},
\end{aligned}
$$

17 
and

$$
\begin{aligned}
\mu_{0} \int_{\mathbb{R}}\left(\partial_{x}^{2} u\right)\left(\partial_{x} u\right) u^{p} g^{\prime \prime} \\
\quad=\frac{\mu_{0}}{2} \int_{\mathbb{R}} \partial_{x}\left[\left(\partial_{x} u\right)^{2}\right] u^{p} g^{\prime \prime} \\
=-\frac{\mu_{0} p}{2} \int_{\mathbb{R}}\left(\partial_{x} u\right)^{3} u^{p-1} g^{\prime \prime}-\frac{\mu_{0}}{2} \int_{\mathbb{R}}\left(\partial_{x} u\right)^{2} u^{p} g^{\prime \prime \prime} .
\end{aligned}
$$

Then, summing (2.109)-2.111), we get

$$
\begin{aligned}
K_{221}= & \mu_{0} \int_{\mathbb{R}}\left(\partial_{x}^{2} u\right)^{2} u^{p} g^{\prime}-\frac{\mu_{0} p(p-1)}{3} \int_{\mathbb{R}}\left(\partial_{x} u\right)^{4} u^{p-2} g^{\prime} \\
& -\frac{5 \mu_{0} p}{6} \int_{\mathbb{R}}\left(\partial_{x} u\right)^{3} u^{p-1} g^{\prime \prime}-\frac{\mu_{0}}{2} \int_{\mathbb{R}}\left(\partial_{x} u\right)^{2} u^{p} g^{\prime \prime \prime} .
\end{aligned}
$$

We check that

$$
\begin{aligned}
K_{222} & =\frac{\mu_{0}}{p+1} \int_{\mathbb{R}}\left(\partial_{x}^{2} u\right) \partial_{x}\left(u^{p+1} g^{\prime \prime}\right) \\
& =\mu_{0} \int_{\mathbb{R}}\left(\partial_{x}^{2} u\right)\left(\partial_{x} u\right) u^{p} g^{\prime \prime}+\frac{\mu_{0}}{p+1} \int_{\mathbb{R}}\left(\partial_{x}^{2} u\right) u^{p+1} g^{\prime \prime \prime},
\end{aligned}
$$

with

$$
\begin{aligned}
\mu_{0} \int_{\mathbb{R}}\left(\partial_{x}^{2} u\right)\left(\partial_{x} u\right) u^{p} g^{\prime \prime} \\
=\frac{\mu_{0}}{2} \int_{\mathbb{R}} \partial_{x}\left(\partial_{x} u\right)^{2} u^{p} g^{\prime \prime} \\
=-\frac{\mu_{0} p}{2} \int_{\mathbb{R}}\left(\partial_{x} u\right)^{3} u^{p-1} g^{\prime \prime}-\frac{\mu_{0}}{2} \int_{\mathbb{R}}\left(\partial_{x} u\right)^{2} u^{p} g^{\prime \prime \prime},
\end{aligned}
$$

and

$$
\begin{aligned}
& \frac{\mu_{0}}{p+1} \int_{\mathbb{R}}\left(\partial_{x}^{2} u\right) u^{p+1} g^{\prime \prime \prime} \\
& \quad=-\frac{\mu_{0}}{p+1} \int_{\mathbb{R}}\left(\partial_{x} u\right) \partial_{x}\left(u^{p+1} g^{\prime \prime \prime}\right) \\
& \quad=-\mu_{0} \int_{\mathbb{R}}\left(\partial_{x} u\right)^{2} u^{p} g^{\prime \prime \prime}-\frac{\mu_{0}}{(p+1)(p+2)} \int_{\mathbb{R}} \partial_{x}\left(u^{p+2}\right) g^{(4)} \\
& \quad=-\mu_{0} \int_{\mathbb{R}}\left(\partial_{x} u\right)^{2} u^{p} g^{\prime \prime \prime}+\frac{\mu_{0}}{(p+1)(p+2)} \int_{\mathbb{R}} u^{p+2} g^{(5)},
\end{aligned}
$$

and then summing 2.114 and 2.115, we get

$$
K_{222}=-\frac{\mu_{0} p}{2} \int_{\mathbb{R}}\left(\partial_{x} u\right)^{3} u^{p-1} g^{\prime \prime}-\frac{3 \mu_{0}}{2} \int_{\mathbb{R}}\left(\partial_{x} u\right)^{2} u^{p} g^{\prime \prime \prime}+\frac{\mu_{0}}{(p+1)(p+2)} \int_{\mathbb{R}} u^{p+2} g^{(5)} \text {. }
$$

Summing 2.112 and 2.116), we get

$$
\begin{aligned}
K_{22}= & \mu_{0} \int_{\mathbb{R}}\left(\partial_{x}^{2} u\right)^{2} u^{p} g^{\prime}-\frac{\mu_{0} p(p-1)}{3} \int_{\mathbb{R}}\left(\partial_{x} u\right)^{4} u^{p-2} g^{\prime}-\frac{4 \mu_{0} p}{3} \int_{\mathbb{R}}\left(\partial_{x} u\right)^{3} u^{p-1} g^{\prime \prime} \\
& -2 \mu_{0} \int_{\mathbb{R}}\left(\partial_{x} u\right)^{2} u^{p} g^{\prime \prime \prime}+\frac{\mu_{0}}{(p+1)(p+2)} \int_{\mathbb{R}} u^{p+2} g^{(5)},
\end{aligned}
$$


and summing 2.107) and 2.117, we get

$$
\begin{aligned}
K_{2}= & \frac{5 \mu_{0} p}{2} \int_{\mathbb{R}}\left(\partial_{x}^{2} u\right)^{2}\left(\partial_{x} u\right) u^{p-1} g-\frac{\mu_{0} p(p-1)(p-2)}{4} \int_{\mathbb{R}}\left(\partial_{x} u\right)^{5} u^{p-3} g+\frac{5 \mu_{0}}{2} \int_{\mathbb{R}}\left(\partial_{x}^{2} u\right)^{2} u^{p} g^{\prime} \\
& -\frac{15 \mu_{0} p(p-1)}{12} \int_{\mathbb{R}}\left(\partial_{x} u\right)^{4} u^{p-2} g^{\prime}-\frac{15 \mu_{0} p}{6} \int_{\mathbb{R}}\left(\partial_{x} u\right)^{3} u^{p-1} g^{\prime \prime} \\
& -\frac{5 \mu_{0}}{2} \int_{\mathbb{R}}\left(\partial_{x} u\right)^{2} u^{p} g^{\prime \prime \prime}+\frac{\mu_{0}}{(p+1)(p+2)} \int_{\mathbb{R}} u^{p+2} g^{(5)}
\end{aligned}
$$

Finally, summing 2.91, 2.95) and 2.118), we obtain

$$
\begin{aligned}
K=\frac{p}{2} \int_{\mathbb{R}}\left(\partial_{x} u\right)^{3} u^{p-1} g+\frac{5 \mu_{0} p}{2} \int_{\mathbb{R}}\left(\partial_{x}^{2} u\right)^{2}\left(\partial_{x} u\right) u^{p-1} g-\frac{\mu_{0} p(p-1)(p-2)}{4} \int_{\mathbb{R}}\left(\partial_{x} u\right)^{5} u^{p-3} g \\
-\frac{1}{2(p+1)^{2}} \int_{\mathbb{R}} u^{2 p+2} g^{\prime}+\frac{3}{2} \int_{\mathbb{R}}\left(\partial_{x} u\right)^{2} u^{p} g^{\prime}-\frac{1}{(p+1)(p+2)} \int_{\mathbb{R}} u^{p+2} g^{\prime \prime \prime} \\
\quad+\frac{5 \mu_{0}}{2} \int_{\mathbb{R}}\left(\partial_{x}^{2} u\right)^{2} u^{p} g^{\prime}-\frac{15 \mu_{0} p(p-1)}{12} \int_{\mathbb{R}}\left(\partial_{x} u\right)^{4} u^{p-2} g^{\prime}-\frac{15 \mu_{0} p}{6} \int_{\mathbb{R}}\left(\partial_{x} u\right)^{3} u^{p-1} g^{\prime \prime} \\
\quad-\frac{5 \mu_{0}}{2} \int_{\mathbb{R}}\left(\partial_{x} u\right)^{2} u^{p} g^{\prime \prime \prime}+\frac{\mu_{0}}{(p+1)(p+2)} \int_{\mathbb{R}} u^{p+2} g^{(5)} .
\end{aligned}
$$

Let us compute $J$ :

$$
J=\int_{\mathbb{R}}\left[\partial_{x}\left(\partial_{t} u\right)\right]\left(\partial_{x} u\right) g
$$

and using that $u(t, x)$ satisfies $\mu_{0}$-gKW equation, we have

$$
\begin{aligned}
J & =-p \int_{\mathbb{R}}\left(\partial_{x} u\right)^{3} u^{p-1} g-\int_{\mathbb{R}}\left(\partial_{x}^{2} u\right)\left(\partial_{x} u\right) u^{p} g-\int_{\mathbb{R}}\left(\partial_{x}^{4} u\right)\left(\partial_{x} u\right) g+\mu_{0} \int_{\mathbb{R}}\left(\partial_{x}^{6} u\right)\left(\partial_{x} u\right) g \\
& =-p \int_{\mathbb{R}}\left(\partial_{x} u\right)^{3} u^{p-1} g+J_{1}+J_{2}+J_{3} .
\end{aligned}
$$

Let us compute $J_{1}$ :

$$
\begin{aligned}
J_{1} & =-\frac{1}{2} \int_{\mathbb{R}} \partial_{x}\left[\left(\partial_{x} u\right)^{2}\right] u^{p} g \\
& =\frac{p}{2} \int_{\mathbb{R}}\left(\partial_{x} u\right)^{3} u^{p-1} g+\frac{1}{2} \int_{\mathbb{R}}\left(\partial_{x} u\right)^{2} u^{p} g^{\prime} .
\end{aligned}
$$

Let us compute $J_{2}$ :

$$
\begin{aligned}
J_{2} & =\int_{\mathbb{R}}\left(\partial_{x}^{3} u\right) \partial_{x}\left[\left(\partial_{x} u\right) g\right] \\
& =\frac{1}{2} \int_{\mathbb{R}} \partial_{x}\left[\left(\partial_{x}^{2} u\right)^{2}\right] g+\int_{\mathbb{R}}\left(\partial_{x}^{3} u\right)\left(\partial_{x} u\right) g^{\prime} \\
& =-\frac{1}{2} \int_{\mathbb{R}}\left(\partial_{x}^{2} u\right)^{2} g^{\prime}+\int_{\mathbb{R}}\left(\partial_{x}^{3} u\right)\left(\partial_{x} u\right) g^{\prime},
\end{aligned}
$$


with

$$
\begin{aligned}
\int_{\mathbb{R}}\left(\partial_{x}^{3} u\right)\left(\partial_{x} u\right) g^{\prime} \\
=-\int_{\mathbb{R}}\left(\partial_{x}^{2} u\right) \partial_{x}\left[\left(\partial_{x} u\right) g^{\prime}\right] \\
=-\int_{\mathbb{R}}\left(\partial_{x}^{2} u\right)^{2} g^{\prime}-\frac{1}{2} \int_{\mathbb{R}} \partial_{x}\left(\partial_{x} u\right)^{2} g^{\prime \prime} \\
=-\int_{\mathbb{R}}\left(\partial_{x}^{2} u\right)^{2} g^{\prime}+\frac{1}{2} \int_{\mathbb{R}}\left(\partial_{x} u\right)^{2} g^{\prime \prime \prime},
\end{aligned}
$$

and then summing 2.123) and 2.124, we get

$$
J_{2}=-\frac{3}{2} \int_{\mathbb{R}}\left(\partial_{x}^{2} u\right)^{2} g^{\prime}+\frac{1}{2} \int_{\mathbb{R}}\left(\partial_{x} u\right)^{2} g^{\prime \prime \prime} .
$$

Let us compute $J_{3}$ :

$$
\begin{aligned}
J_{3} & =-\mu_{0} \int_{\mathbb{R}}\left(\partial_{x}^{5} u\right) \partial_{x}\left[\left(\partial_{x} u\right) g\right] \\
& =-\mu_{0} \int_{\mathbb{R}}\left(\partial_{x}^{5} u\right)\left(\partial_{x}^{2} u\right) g-\mu_{0} \int_{\mathbb{R}}\left(\partial_{x}^{5} u\right)\left(\partial_{x} u\right) g^{\prime}=J_{31}+J_{32} .
\end{aligned}
$$

Let us compute $J_{31}$ :

$$
\begin{aligned}
J_{31} & =\mu_{0} \int_{\mathbb{R}}\left(\partial_{x}^{4} u\right) \partial_{x}\left[\left(\partial_{x}^{2} u\right) g\right] \\
& =\frac{\mu_{0}}{2} \int_{\mathbb{R}} \partial_{x}\left[\left(\partial_{x}^{3} u\right)^{2}\right] g+\mu_{0} \int_{\mathbb{R}}\left(\partial_{x}^{4} u\right)\left(\partial_{x}^{2} u\right) g^{\prime} \\
& =-\frac{\mu_{0}}{2} \int_{\mathbb{R}}\left(\partial_{x}^{3} u\right)^{2} g^{\prime}+\mu_{0} \int_{\mathbb{R}}\left(\partial_{x}^{4} u\right)\left(\partial_{x}^{2} u\right) g^{\prime},
\end{aligned}
$$

with

$$
\begin{aligned}
\mu_{0} \int_{\mathbb{R}}\left(\partial_{x}^{4} u\right)\left(\partial_{x}^{2} u\right) g^{\prime} \\
\quad=-\mu_{0} \int_{\mathbb{R}}\left(\partial_{x}^{3} u\right) \partial_{x}\left[\left(\partial_{x}^{2} u\right) g^{\prime}\right] \\
\quad=-\mu_{0} \int_{\mathbb{R}}\left(\partial_{x}^{3} u\right)^{2} g^{\prime}-\frac{\mu_{0}}{2} \int_{\mathbb{R}} \partial_{x}\left[\left(\partial_{x}^{2} u\right)^{2}\right] g^{\prime \prime} \\
\quad=-\mu_{0} \int_{\mathbb{R}}\left(\partial_{x}^{3} u\right)^{2} g^{\prime}+\frac{\mu_{0}}{2} \int_{\mathbb{R}}\left(\partial_{x}^{2} u\right)^{2} g^{\prime \prime \prime},
\end{aligned}
$$

and then summing (2.127) and (2.128), we get

$$
J_{31}=-\frac{3 \mu_{0}}{2} \int_{\mathbb{R}}\left(\partial_{x}^{3} u\right)^{2} g^{\prime}+\frac{\mu_{0}}{2} \int_{\mathbb{R}}\left(\partial_{x}^{2} u\right)^{2} g^{\prime \prime \prime} .
$$

Let us compute $J_{32}$ :

$$
\begin{aligned}
J_{32} & =\mu_{0} \int_{\mathbb{R}}\left(\partial_{x}^{4} u\right) \partial_{x}\left[\left(\partial_{x} u\right) g^{\prime}\right] \\
& =\mu_{0} \int_{\mathbb{R}}\left(\partial_{x}^{4} u\right)\left(\partial_{x}^{2} u\right) g^{\prime}+\mu_{0} \int_{\mathbb{R}}\left(\partial_{x}^{4} u\right)\left(\partial_{x} u\right) g^{\prime \prime}=J_{321}+J_{322},
\end{aligned}
$$


with

$$
\begin{aligned}
J_{321} & =-\mu_{0} \int_{\mathbb{R}}\left(\partial_{x}^{3} u\right) \partial_{x}\left[\left(\partial_{x}^{2} u\right) g^{\prime}\right] \\
& =-\mu_{0} \int_{\mathbb{R}}\left(\partial_{x}^{3} u\right)^{2} g^{\prime}-\frac{\mu_{0}}{2} \int_{\mathbb{R}} \partial_{x}\left[\left(\partial_{x}^{2} u\right)^{2}\right] g^{\prime \prime} \\
& =-\mu_{0} \int_{\mathbb{R}}\left(\partial_{x}^{3} u\right)^{2} g^{\prime}+\frac{\mu_{0}}{2} \int_{\mathbb{R}}\left(\partial_{x}^{2} u\right)^{2} g^{\prime \prime \prime}
\end{aligned}
$$

and

$$
\begin{aligned}
J_{322} & =-\mu_{0} \int_{\mathbb{R}}\left(\partial_{x}^{3} u\right) \partial_{x}\left[\left(\partial_{x} u\right) g^{\prime \prime}\right] \\
& =-\frac{\mu_{0}}{2} \int_{\mathbb{R}} \partial_{x}\left[\left(\partial_{x}^{2} u\right)^{2}\right] g^{\prime \prime}-\mu_{0} \int_{\mathbb{R}}\left(\partial_{x}^{3} u\right)\left(\partial_{x} u\right) g^{\prime \prime \prime} \\
& =\frac{\mu_{0}}{2} \int_{\mathbb{R}}\left(\partial_{x}^{2} u\right)^{2} g^{\prime \prime \prime}-\mu_{0} \int_{\mathbb{R}}\left(\partial_{x}^{3} u\right)\left(\partial_{x} u\right) g^{\prime \prime \prime},
\end{aligned}
$$

with

$$
\begin{aligned}
& -\mu_{0} \int_{\mathbb{R}}\left(\partial_{x}^{3} u\right)\left(\partial_{x} u\right) g^{\prime \prime \prime} \\
& \quad=\mu_{0} \int_{\mathbb{R}}\left(\partial_{x}^{2} u\right) \partial_{x}\left[\left(\partial_{x} u\right) g^{\prime \prime \prime}\right] \\
& \quad=\mu_{0} \int_{\mathbb{R}}\left(\partial_{x}^{2} u\right)^{2} g^{\prime \prime \prime}+\frac{\mu_{0}}{2} \int_{\mathbb{R}} \partial_{x}\left[\left(\partial_{x} u\right)^{2}\right] g^{(4)} \\
& \quad=\mu_{0} \int_{\mathbb{R}}\left(\partial_{x}^{2} u\right)^{2} g^{\prime \prime \prime}-\frac{\mu_{0}}{2} \int_{\mathbb{R}}\left(\partial_{x} u\right)^{2} g^{(5)}
\end{aligned}
$$

and summing 2.132 and 2.133 , we get

$$
J_{322}=\frac{3 \mu_{0}}{2} \int_{\mathbb{R}}\left(\partial_{x}^{2} u\right)^{2} g^{\prime \prime \prime}-\frac{\mu_{0}}{2} \int_{\mathbb{R}}\left(\partial_{x} u\right)^{2} g^{(5)} .
$$

Then, summing 2.131) and 2.134, we get

$$
J_{32}=-\mu_{0} \int_{\mathbb{R}}\left(\partial_{x}^{3} u\right)^{2} g^{\prime}+2 \mu_{0} \int_{\mathbb{R}}\left(\partial_{x}^{2} u\right)^{2} g^{\prime \prime \prime}-\frac{\mu_{0}}{2} \int_{\mathbb{R}}\left(\partial_{x} u\right)^{2} g^{(5)},
$$

and summing 2.129) and 2.135, we get

$$
J_{3}=-\frac{5 \mu_{0}}{2} \int_{\mathbb{R}}\left(\partial_{x}^{3} u\right)^{2} g^{\prime}+\frac{5 \mu_{0}}{2} \int_{\mathbb{R}}\left(\partial_{x}^{2} u\right)^{2} g^{\prime \prime \prime}-\frac{\mu_{0}}{2} \int_{\mathbb{R}}\left(\partial_{x} u\right)^{2} g^{(5)} .
$$

Finally, summing 2.121, 2.122, 2.125 and 2.136, we obtain

$$
\begin{aligned}
J= & -\frac{p}{2} \int_{\mathbb{R}}\left(\partial_{x} u\right)^{3} u^{p-1} g+\frac{1}{2} \int_{\mathbb{R}}\left(\partial_{x} u\right)^{2} u^{p} g^{\prime}-\frac{3}{2} \int_{\mathbb{R}}\left(\partial_{x}^{2} u\right)^{2} g^{\prime}+\frac{1}{2} \int_{\mathbb{R}}\left(\partial_{x} u\right)^{2} g^{\prime \prime \prime} \\
& -\frac{5 \mu_{0}}{2} \int_{\mathbb{R}}\left(\partial_{x}^{3} u\right)^{2} g^{\prime}+\frac{5 \mu_{0}}{2} \int_{\mathbb{R}}\left(\partial_{x}^{2} u\right)^{2} g^{\prime \prime \prime}-\frac{\mu_{0}}{2} \int_{\mathbb{R}}\left(\partial_{x} u\right)^{2} g^{(5)} .
\end{aligned}
$$

Let us compute $I$ :

$$
I=\mu_{0} \int_{\mathbb{R}}\left[\partial_{x}^{2}\left(\partial_{t} u\right)\right]\left(\partial_{x}^{2} u\right) g
$$


and using that $u(t, x)$ satisfies $\mu_{0}$-gKW equation, we have

$$
\begin{aligned}
I= & -3 p \mu_{0} \int_{\mathbb{R}}\left(\partial_{x}^{2} u\right)^{2}\left(\partial_{x} u\right) u^{p-1} g-\mu_{0} p(p-1) \int_{\mathbb{R}}\left(\partial_{x}^{2} u\right)\left(\partial_{x} u\right)^{3} u^{p-2} g-\mu_{0} \int_{\mathbb{R}}\left(\partial_{x}^{3} u\right)\left(\partial_{x}^{2} u\right) u^{p} g \\
& -\mu_{0} \int_{\mathbb{R}}\left(\partial_{x}^{5} u\right)\left(\partial_{x}^{2} u\right) g+\mu_{0}^{2} \int_{\mathbb{R}}\left(\partial_{x}^{7} u\right)\left(\partial_{x}^{2} u\right) g \\
= & -3 p \mu_{0} \int_{\mathbb{R}}\left(\partial_{x}^{2} u\right)^{2}\left(\partial_{x} u\right) u^{p-1} g+\sum_{j=1}^{4} I_{j} .
\end{aligned}
$$

Let us compute $I_{1}$ :

$$
\begin{aligned}
I_{1} & =-\frac{\mu_{0} p(p-1)}{4} \int_{\mathbb{R}} \partial_{x}\left[\left(\partial_{x} u\right)^{4}\right] u^{p-2} g \\
& =\frac{\mu_{0} p(p-1)(p-2)}{4} \int_{\mathbb{R}}\left(\partial_{x} u\right)^{5} u^{p-3} g+\frac{\mu_{0} p(p-1)}{4} \int_{\mathbb{R}}\left(\partial_{x} u\right)^{4} u^{p-2} g^{\prime} .
\end{aligned}
$$

Let us compute $I_{2}$ :

$$
\begin{aligned}
I_{2} & =-\frac{\mu_{0}}{2} \int_{\mathbb{R}} \partial_{x}\left[\left(\partial_{x}^{2} u\right)^{2}\right] u^{p} g \\
& =\frac{\mu_{0} p}{2} \int_{\mathbb{R}}\left(\partial_{x}^{2} u\right)^{2}\left(\partial_{x} u\right) u^{p-1} g+\frac{\mu_{0}}{2} \int_{\mathbb{R}}\left(\partial_{x}^{2} u\right)^{2} u^{p} g^{\prime} .
\end{aligned}
$$

Let us compute $I_{3}$ :

$$
\begin{aligned}
I_{3} & =\mu_{0} \int_{\mathbb{R}}\left(\partial_{x}^{4} u\right) \partial_{x}\left[\left(\partial_{x}^{2} u\right) g\right] \\
& =\frac{\mu_{0}}{2} \int_{\mathbb{R}} \partial_{x}\left[\left(\partial_{x}^{3} u\right)^{2}\right] g+\mu_{0} \int_{\mathbb{R}}\left(\partial_{x}^{4} u\right)\left(\partial_{x}^{2} u\right) g^{\prime} \\
& =-\frac{\mu_{0}}{2} \int_{\mathbb{R}}\left(\partial_{x}^{3} u\right)^{2} g^{\prime}+I_{31},
\end{aligned}
$$

with

$$
\begin{aligned}
I_{31} & =-\mu_{0} \int_{\mathbb{R}}\left(\partial_{x}^{3} u\right) \partial_{x}\left[\left(\partial_{x}^{2} u\right) g^{\prime}\right] \\
& =-\mu_{0} \int_{\mathbb{R}}\left(\partial_{x}^{3} u\right)^{2} g^{\prime}-\frac{\mu_{0}}{2} \int_{\mathbb{R}} \partial_{x}\left[\left(\partial_{x}^{2} u\right)^{2}\right] g^{\prime \prime} \\
& =-\mu_{0} \int_{\mathbb{R}}\left(\partial_{x}^{3} u\right)^{2} g^{\prime}+\frac{\mu_{0}}{2} \int_{\mathbb{R}}\left(\partial_{x}^{2} u\right)^{2} g^{\prime \prime \prime}
\end{aligned}
$$

and summing 2.142 and 2.143, we get

$$
I_{3}=-\frac{3 \mu_{0}}{2} \int_{\mathbb{R}}\left(\partial_{x}^{3} u\right)^{2} g^{\prime}+\frac{\mu_{0}}{2} \int_{\mathbb{R}}\left(\partial_{x}^{2} u\right)^{2} g^{\prime \prime \prime} .
$$

Let us compute $I_{4}$ :

$$
\begin{aligned}
I_{4} & =-\mu_{0}^{2} \int_{\mathbb{R}}\left(\partial_{x}^{6} u\right) \partial_{x}\left[\left(\partial_{x}^{2} u\right) g\right] \\
& =-\mu_{0}^{2} \int_{\mathbb{R}}\left(\partial_{x}^{6} u\right)\left(\partial_{x}^{3} u\right) g-\mu_{0}^{2} \int_{\mathbb{R}}\left(\partial_{x}^{6} u\right)\left(\partial_{x}^{2} u\right) g^{\prime}=I_{41}+I_{42} .
\end{aligned}
$$


Let us compute $I_{41}$ :

$$
\begin{aligned}
I_{41} & =\mu_{0}^{2} \int_{\mathbb{R}}\left(\partial_{x}^{5} u\right) \partial_{x}\left[\left(\partial_{x}^{3} u\right) g\right] \\
& =\frac{\mu_{0}^{2}}{2} \int_{\mathbb{R}} \partial_{x}\left[\left(\partial_{x}^{4} u\right)^{2}\right] g+\mu_{0}^{2} \int_{\mathbb{R}}\left(\partial_{x}^{5} u\right)\left(\partial_{x}^{3} u\right) g^{\prime} \\
& =-\frac{\mu_{0}^{2}}{2} \int_{\mathbb{R}}\left(\partial_{x}^{4} u\right)^{2} g^{\prime}+I_{411},
\end{aligned}
$$

with

$$
\begin{aligned}
I_{411} & =-\mu_{0}^{2} \int_{\mathbb{R}}\left(\partial_{x}^{4} u\right) \partial_{x}\left[\left(\partial_{x}^{3} u\right) g^{\prime}\right] \\
& =-\mu_{0}^{2} \int_{\mathbb{R}}\left(\partial_{x}^{4} u\right)^{2} g^{\prime}-\frac{\mu_{0}^{2}}{2} \int_{\mathbb{R}} \partial_{x}\left[\left(\partial_{x}^{3} u\right)^{2}\right] g^{\prime \prime} \\
& =-\mu_{0}^{2} \int_{\mathbb{R}}\left(\partial_{x}^{4} u\right)^{2} g^{\prime}+\frac{\mu_{0}^{2}}{2} \int_{\mathbb{R}}\left(\partial_{x}^{3} u\right)^{2} g^{\prime \prime \prime},
\end{aligned}
$$

and summing 2.146 and 2.147, we get

$$
I_{41}=-\frac{3 \mu_{0}^{2}}{2} \int_{\mathbb{R}}\left(\partial_{x}^{4} u\right)^{2} g^{\prime}+\frac{\mu_{0}^{2}}{2} \int_{\mathbb{R}}\left(\partial_{x}^{3} u\right)^{2} g^{\prime \prime \prime} .
$$

Let us compute $I_{42}$ :

$$
\begin{aligned}
I_{42} & =\mu_{0}^{2} \int_{\mathbb{R}}\left(\partial_{x}^{5} u\right) \partial_{x}\left[\left(\partial_{x}^{2} u\right) g^{\prime}\right] \\
& =\mu_{0}^{2} \int_{\mathbb{R}}\left(\partial_{x}^{5} u\right)\left(\partial_{x}^{3} u\right) g^{\prime}+\mu_{0}^{2} \int_{\mathbb{R}}\left(\partial_{x}^{5} u\right)\left(\partial_{x}^{2} u\right) g^{\prime \prime}=I_{421}+I_{422} .
\end{aligned}
$$

Let us compute $I_{421}$ :

$$
\begin{aligned}
I_{421} & =-\mu_{0}^{2} \int_{\mathbb{R}}\left(\partial_{x}^{4} u\right) \partial_{x}\left[\left(\partial_{x}^{3} u\right) g^{\prime}\right] \\
& =-\mu_{0}^{2} \int_{\mathbb{R}}\left(\partial_{x}^{4} u\right)^{2} g^{\prime}-\frac{\mu_{0}^{2}}{2} \int_{\mathbb{R}} \partial_{x}\left[\left(\partial_{x}^{3} u\right)^{2}\right] g^{\prime \prime} \\
& =-\mu_{0}^{2} \int_{\mathbb{R}}\left(\partial_{x}^{4} u\right)^{2} g^{\prime}+\frac{\mu_{0}^{2}}{2} \int_{\mathbb{R}}\left(\partial_{x}^{3} u\right)^{2} g^{\prime \prime \prime}
\end{aligned}
$$

Let us compute $I_{422}$ :

$$
\begin{aligned}
I_{422} & =-\mu_{0}^{2} \int_{\mathbb{R}}\left(\partial_{x}^{4} u\right) \partial_{x}\left[\left(\partial_{x}^{2} u\right) g^{\prime \prime}\right] \\
& =-\frac{\mu_{0}^{2}}{2} \int_{\mathbb{R}} \partial_{x}\left[\left(\partial_{x}^{3} u\right)^{2}\right] g^{\prime \prime}-\mu_{0}^{2} \int_{\mathbb{R}}\left(\partial_{x}^{4} u\right)\left(\partial_{x}^{2} u\right) g^{\prime \prime \prime} \\
& =\frac{\mu_{0}^{2}}{2} \int_{\mathbb{R}}\left(\partial_{x}^{3} u\right)^{2} g^{\prime \prime \prime}+I_{4221},
\end{aligned}
$$

with

$$
\begin{aligned}
I_{4221} & =\mu_{0}^{2} \int_{\mathbb{R}}\left(\partial_{x}^{3} u\right) \partial_{x}\left[\left(\partial_{x}^{2} u\right) g^{\prime \prime \prime}\right] \\
& =\mu_{0}^{2} \int_{\mathbb{R}}\left(\partial_{x}^{3} u\right)^{2} g^{\prime \prime \prime}+\frac{\mu_{0}^{2}}{2} \int_{\mathbb{R}} \partial_{x}\left[\left(\partial_{x}^{2} u\right)^{2}\right] g^{(4)} \\
& =\mu_{0}^{2} \int_{\mathbb{R}}\left(\partial_{x}^{3} u\right)^{2} g^{\prime \prime \prime}-\frac{\mu_{0}^{2}}{2} \int_{\mathbb{R}}\left(\partial_{x}^{2} u\right)^{2} g^{(5)},
\end{aligned}
$$


and summing 2.151) and 2.152, we get

$$
I_{422}=\frac{3 \mu_{0}^{2}}{2} \int_{\mathbb{R}}\left(\partial_{x}^{3} u\right)^{2} g^{\prime \prime \prime}-\frac{\mu_{0}^{2}}{2} \int_{\mathbb{R}}\left(\partial_{x}^{2} u\right)^{2} g^{(5)} .
$$

Then, summing 2.150 and 2.153, we get

$$
I_{42}=-\mu_{0}^{2} \int_{\mathbb{R}}\left(\partial_{x}^{4} u\right)^{2} g^{\prime}+2 \mu_{0}^{2} \int_{\mathbb{R}}\left(\partial_{x}^{3} u\right)^{2} g^{\prime \prime \prime}-\frac{\mu_{0}^{2}}{2} \int_{\mathbb{R}}\left(\partial_{x}^{2} u\right)^{2} g^{(5)} .
$$

Now, summing 2.148] and 2.154, we get

$$
I_{4}=-\frac{5 \mu_{0}^{2}}{2} \int_{\mathbb{R}}\left(\partial_{x}^{4} u\right)^{2} g^{\prime}+\frac{5 \mu_{0}^{2}}{2} \int_{\mathbb{R}}\left(\partial_{x}^{3} u\right)^{2} g^{\prime \prime \prime}-\frac{\mu_{0}^{2}}{2} \int_{\mathbb{R}}\left(\partial_{x}^{2} u\right)^{2} g^{(5)} .
$$

Finally, we obtain

$$
\begin{aligned}
I=- & \frac{5 \mu_{0} p}{2} \int_{\mathbb{R}}\left(\partial_{x}^{2} u\right)^{2}\left(\partial_{x} u\right) u^{p-1} g+\frac{\mu_{0} p(p-1)(p-2)}{4} \int_{\mathbb{R}}\left(\partial_{x} u\right)^{5} u^{p-3} g+\frac{\mu_{0} p(p-1)}{4} \int_{\mathbb{R}}\left(\partial_{x} u\right)^{4} u^{p-2} g^{\prime} \\
+ & \frac{\mu_{0}}{2} \int_{\mathbb{R}}\left(\partial_{x}^{2} u\right)^{2} u^{p} g^{\prime}-\frac{3 \mu_{0}}{2} \int_{\mathbb{R}}\left(\partial_{x}^{3} u\right)^{2} g^{\prime}+\frac{\mu_{0}}{2} \int_{\mathbb{R}}\left(\partial_{x}^{2} u\right)^{2} g^{\prime \prime \prime} \\
& \quad-\frac{5 \mu_{0}^{2}}{2} \int_{\mathbb{R}}\left(\partial_{x}^{4} u\right)^{2} g^{\prime}+\frac{5 \mu_{0}^{2}}{2} \int_{\mathbb{R}}\left(\partial_{x}^{3} u\right)^{2} g^{\prime \prime \prime}-\frac{\mu_{0}^{2}}{2} \int_{\mathbb{R}}\left(\partial_{x}^{2} u\right)^{2} g^{(5)} .
\end{aligned}
$$

Identity 2.88) follows by combining 2.119, 2.137) and 2.156.

Acknowledgements. The pulication has been prepared with the support of the RUDN University Program 5-100. 


\section{References}

[1] L. Abdelouhab, J. L. Bona, M. Felland, and J.-C. Saut. Nonlocal models for nonlinear, dispersive waves. Phys. D, 40(3):360-392, 1989.

[2] B. Dey, A. Khare, and C. N. Kumar. Stationary solitons of the fifth order KdV-type. Equations and their stabilization. Phys. Lett. A, 223(6):449-452, 1996.

[3] André Kabakouala. A Linear Liouville Property for the Generalized Kawahara Equation. working paper or preprint, October 2018.

[4] André Kabakouala and Luc Molinet. On the stability of the solitary waves to the (generalized) Kawahara equation. J. Math. Anal. Appl., 457(1):478-497, 2018.

[5] V. I. Karpman. Stabilization of soliton instabilities by higher order dispersion: KdV-type equations. Phys. Lett. A, 210(1-2):77-84, 1996.

[6] Celine Laurent and Yvan Martel. Smoothness and exponential decay of $L^{2}$-compact solutions of the generalized KdV equations. Comm. Partial Differential Equations, 28(11-12):2093-2107, 2003.

[7] Yvan Martel. Linear problems related to asymptotic stability of solitons of the generalized KdV equations. SIAM J. Math. Anal., 38(3):759-781, 2006.

[8] Yvan Martel and Frank Merle. Asymptotic stability of solitons for subcritical generalized KdV equations. Arch. Ration. Mech. Anal., 157(3):219-254, 2001.

[9] Yvan Martel and Frank Merle. Asymptotic stability of solitons of the gKdV equations with general nonlinearity. Math. Ann., 341(2):391-427, 2008. 